\title{
The translocator protein (TSPO) is prodromal to mitophagy loss in neurotoxicity
}

\author{
Michele Frison $^{1,9} \cdot$ Danilo Faccenda $^{1} \cdot$ Rosella Abeti $^{2} \cdot$ Manuel Rigon $^{1,3} \cdot$ Daniela Strobbe $^{3} \cdot$ \\ Britannie S. England-Rendon ${ }^{1} \cdot$ Diana Cash $\mathbb{D}^{4} \cdot{\text { Katy } \text { Barnes }^{5} \cdot \text { Mona Sadeghian }^{6} \cdot \text { Marija Sajic }^{6}{ }^{6} \cdot \text { Lisa A. Wells }}^{7}$. \\ Dong $\mathrm{Xia}^{1} \cdot$ Paola Giunti ${ }^{2} \cdot$ Kenneth $\mathrm{Smith}^{6} \cdot$ Heather Mortiboys ${ }^{5} \cdot$ Federico E. Turkheimer $\mathbb{B}^{4}$. \\ Michelangelo Campanella $\mathbb{I D}^{1,3,8}$
}

Received: 10 July 2020 / Revised: 13 January 2021 / Accepted: 5 February 2021 / Published online: 4 March 2021

(c) The Author(s), under exclusive licence to Springer Nature Limited 2021. This article is published with open access

\begin{abstract}
Dysfunctional mitochondria characterise Parkinson's Disease (PD). Uncovering etiological molecules, which harm the homeostasis of mitochondria in response to pathological cues, is therefore pivotal to inform early diagnosis and therapy in the condition, especially in its idiopathic forms. This study proposes the $18 \mathrm{kDa}$ Translocator Protein (TSPO) to be one of those. Both in vitro and in vivo data show that neurotoxins, which phenotypically mimic PD, increase TSPO to enhance cellular redox-stress, susceptibility to dopamine-induced cell death, and repression of ubiquitin-dependent mitophagy. TSPO amplifies the extracellular signal-regulated protein kinase 1 and 2 (ERK1/2) signalling, forming positive feedback, which represses the transcription factor EB (TFEB) and the controlled production of lysosomes. Finally, genetic variances in the transcriptome confirm that TSPO is required to alter the autophagy-lysosomal pathway during neurotoxicity.
\end{abstract}

\section{Introduction}

Prevalence of psychiatric symptoms in primary mitochondrial diseases are common [1,2] and debilitating [3]. Secondary mitochondrial diseases are equally affected, and Parkinsonism is a vastly acknowledged example [4-7]. Centred around a progressive age-related loss of dopaminergic circuits -responsible for bradykinesia and tremor-,

Supplementary information The online version contains supplementary material available at https://doi.org/10.1038/s41380021-01050-z.

$\triangle$ Michelangelo Campanella

mcampanella@rvc.ac.uk

1 Department of Comparative Biomedical Sciences, The Royal Veterinary College, University of London, Royal College Street, London, United Kingdom

2 Ataxia Centre, Department of Clinical and Movement Neurosciences, UCL Queen Square Institute of Neurology, Queen Square London, United Kingdom

3 Department of Biology, University of Rome TorVergata, Via della Ricerca Scientifica, Rome, Italy

4 Department of Neuroimaging, Institute of Psychiatry, King's
Parkinsonism and PD display non-motor features ranging from depression, gambling addiction, and dementia. The symptomatology of PD varies substantially between patients, with mechanisms that remain ill-defined. One useful tool to insight those and stratify patients from a mitochondrial perspective is the Translocator Protein (TSPO) $[8,9]$. This multidrug-binding protein, which is resident on the Outer Mitochondrial Membrane (OMM), has been studied for over two decades as a diagnostic marker of neurotrauma and target for anxiolytic therapy $[10,11]$. Recently, evidence demonstrating the increased TSPO ligand binding in PD patients [12] made us query whether the protein had a causal role in the mitochondrial

College London, Camberwell, United Kingdom

5 Sheffield Institute for Translational Neuroscience (SITraN), University of Sheffield, Sheffield, United Kingdom

6 Department of Neuroinflammation, UCL Queen Square Institute of Neurology, London, United Kingdom

7 Imanova Limited, Centre for Imaging Sciences, Imperial College London, Hammersmith Hospital, London, United Kingdom

8 University College London Consortium for Mitochondrial Research, London, United Kingdom

9 Present address: MRC Mitochondrial Biology Unit, Cambridge Biomedical Campus, Cambridge, United Kingdom 
dysfunction underlying the condition. Clinical positron emission tomography (PET) imaging of patients with PD, dementia with Lewy bodies [13], and progressive supranuclear palsy [14] have revealed a complex but clinically valuable positive correlation between TSPO and the heterogeneous neurological damage [12]. Even though basally expressed at high levels only within steroid producing tissues $[15,16]$, TSPO is promptly inducible in cell lineages of the immune systems, including those residents in the CNS such as microglia [17-19]. In those, neurological stress induces a rapid and localised increase in TSPO [20-22]. However, the same occurs in neurons [18, 23-27], measurable via its targeting compounds [8, 19, 28, 29].

Previously we reported that TSPO prevents mitochondrial ubiquitination and impairs mitophagy [30], speculating that this anti-mitophagy function could be replicated in diseases. Even though PD is multifactorial, the accumulation of defective mitochondria is considered one of its hallmark [31, 32] driven by impairments in their autophagic degradation [7, 33, 34]. Studies of the hereditary forms of $\mathrm{PD}$ have indeed unveiled mutations in PTEN-induced kinase 1 (PINK1) [35] and the RBR E3 ubiquitin protein ligase Parkin (PARK2) genes [36], associated with a failure of mitochondrial autophagy as one of the causative mechanisms [37-39]. However, the PINK1-Parkin pathway of mitophagy requires potent toxins, like ETC uncouplers (e.g. FCCP), to be observed in vitro while disposable for mitophagy in vivo [40, 41]. In addition, recent studies have suggested physiological functions for the PINK1-Parkin pathway, which go beyond mitophagy, such as inflammation [42, 43] and regulation of mitochondrial protein import [44-46]. The selection and isolation of damaged mitochondria from the network require the essential ubiquitination of OMM proteins to consent their removal via the autophagosomal-lysosomal pathway [34, 38, 47, 48].

Based on this evidence, we set out to probe whether neurotoxin-induced mitochondrial damage is associated with the upregulation of TSPO and deregulation of mitophagy. We did so aware that: (i) TSPO expression is driven by activation of the extracellular signal-regulated protein kinases (ERKs) [49], and (ii) the mitogen-activated protein kinase (MAPK)/ERK signalling pathway is a relevant event during neurotoxicity and particularly PD [50]. The phosphorylated form (pERK1/2) is indeed found in Lewy Body aggregates and autophagosomes of idiopathic PD patients [51]. Parkinsonian neurotoxins [6-OHDA, rotenone, and Nmethyl-4-phenylpyridinium (MPP+) particularly] induce ERK phosphorylation following an increase in Reactive Oxygen Species (ROS), which actively confer neurotoxicity by inducing cytotoxic autophagy [52] and impairing mitochondrial biogenesis [53]. Therefore, activated ERK may play a role in mediating mitochondrial dysfunction during
PD-like neuronal injury [54] and TSPO high expression which is indeed driven by ERK [8].

Here, we demonstrate that TSPO is a factor in the mitochondrial aetiology of neurotoxicity by operating at multiple levels to impair the autophagosome-lysosomal pathway triggered by PD toxins. Therefore, TSPO emerges as a pro-pathological conduit for mitochondria, which results in several distinctive signatures of the transcriptome.

\section{Materials and methods}

\section{Animal experiments}

6-OHDA lesioned rat brain sections for immunohistological analysis were prepared as previously described [55]. Briefly, the rats (male, Sprague Dawley, 250 $\pm 25 \mathrm{~g}$, Harlan, UK) were group-housed at standard conditions, and all experiments were conducted under the Home Office Animals (Scientific procedures) Act, UK, 1986 and were approved by the King's College London ethical review committee (project licence P023CC39A, D Cash). The lesioning with 6OHDA was achieved by stereotaxically administering $12 \mu \mathrm{g}$ of 6-OHDA into the medial forebrain bundle. The success of lesioning was verified by apomorphine-induced rotations (>100 in $60 \mathrm{~min}$ ), and the animals sacrificed 3 weeks after 6OHDA by transcardiac perfusion and fixation with heparinised saline, followed by $4 \%$ buffered paraformaldehyde. PET imaging was carried out on a separate set of animals in a separate facility. Male Sprague-Dawley rats (Charles River, UK) weighing ca. $250 \mathrm{~g}$, were housed in groups of three per cage under a controlled temperature $\left(21+1^{\circ} \mathrm{C}\right)$, relative humidity $(60 \%)$ and a $12 \mathrm{~h}$ light/dark cycle, with access to food and water ad libitum.

\section{Surgery and imaging}

Under recovery anaesthesia (isoflurane), rats were injected with 6-OHDA. Twelve $\mu$ g 6-OHDA free base in $4 \mu \mathrm{l} 0.1 \%$ ascorbic acid in $0.9 \%$ saline $(n=4)$ or vehicle (control $n=2$ ) was injected stereotactically [56] into the left medial forebrain bundle $(\mathrm{mfb})(-2.2 \mathrm{~mm} \mathrm{~A} / \mathrm{P}, \quad+1.5 \mathrm{~mm} \mathrm{~L} / \mathrm{M}$ coordinates from bregma; $-7.9 \mathrm{~mm} \mathrm{D} / \mathrm{V}$ from dura), as previously described by the authors [57]. Nine days postlesion rats received an i.v. bolus of $\left[{ }^{11} \mathrm{C}\right] \mathrm{PBR} 28$. Subsequently, two of the four lesioned rats underwent a PET:CT scan. $\left[{ }^{11} \mathrm{C}\right] \mathrm{PBR} 28$ was prepared by ${ }^{11} \mathrm{C}$-methylation of the corresponding $O$-desmethyl precursor using $\left[{ }^{11} \mathrm{C}\right]$ methyl iodide. The specific activity was $289 \mathrm{~GB}$ q/umol. For imaging studies, the femoral vein and artery of SD rats were cannulated under terminal isoflurane anaesthesia prior to $\left[{ }^{11}\right.$ C]PBR28 administration. A dynamic PET:CT scan 
(INVEON DPET/MM PET:CT, $60 \mathrm{~min}$ ) was carried out and reconstructed with $2 \mathrm{D}$ FBP (CT attenuated with scatter correction). Blood samples were collected to obtain a metabolite-corrected input function. Regions of interest (ROI) were drawn on the lesion site and an equivalent contralateral area. Time activity curves (TACs) were derived, and the volume of distribution $\left(\mathrm{V}_{\mathrm{T}}\right)$ was estimated. After $60 \mathrm{~min}$, all rats were exsanguinated; blood and plasma were collected, and brain regions were removed and washed in chilled saline, and the associated radioactivity was determined in all samples by a gamma counter. For $\left[{ }^{3} \mathrm{H}\right]$ PBR28 radiography, striatal and substantia nigral sections (4.52-4.80 mm from Bregma) were cut onto poly-l-lysine coated slides and incubated with $0.5 \mathrm{nM}$ [3H]PBR28 for $60 \mathrm{~min}$. To determine non-specific binding, slides were incubated with $0.5 \mathrm{nM}$ [3H]PBR28 with an excess of $10 \mu \mathrm{M}$ PK11195 for $60 \mathrm{~min}$. Slides were washed and allowed to dry in a cool stream of air. Slides were covered by a scintillation sheet and counted for $24 \mathrm{~h}$ per slide. Quantification of the autoradiography images was carried out using ImageJ, by comparing the values extracted from each ipsilateral hemisphere to respective contralateral Control. Each image was separately assessed using the Max Entropy algorithm in the area of pixels above threshold.

\section{Cell culture and transfection}

SH-SY5Y cells were obtained from ATCC and were maintained in Dulbecco's Modified Eagle's medium (DMEM) (Thermo Fisher, 11995073) supplemented with 10\% Foetal bovine serum (FBS) (Thermo Fisher, 10082-147) and 1\% penicillin/streptomycin (P/S) (Thermo Fisher, 15140122). To induce differentiation, a serial treatment combination of reduced medium (DMEM $+0.5 \% \mathrm{FBS}$ and $1 \% \mathrm{P} / \mathrm{S})$ for 10 days, with the first 3 days in $15 \mu \mathrm{M}$ retinoic acid (RA; Sigma, R2625) and the successive 7 days of $0.5 \mathrm{ng} / \mathrm{mL}$ BDNF (Sigma, B3795). Transfection was carried out using a standard Calcium $\left(\mathrm{Ca}^{2+}\right)$ phosphate method, as described previously [58]. In experiments containing +TSPO and -TSPO conditions together, the RNAi samples (nsc and -TSPO) were normalised to the mean of the mock transfected samples. Treatments were initiated $48 \mathrm{~h}$ post-transfection. Plasmid transfection was used to induce expression of exogenous proteins, over-expression of selected proteins or downregulation of TSPO via RNA interference (SI02777061, Qiagen; sense: CAUCUUCUUUGGUGCCCGATT; antisense: UCGGGCACCAAAGAAGAUGGG). Plasmid list: scrambled RNA (scrRNA) (1027281, Qiagen), non-silencing control (nsc); mTSPO - pCR $^{\mathrm{TM}}$ 2.1-TOPO ${ }^{\circledR}$ TA vector with gene D21027, Parkin-YFP (Addgene, 23955), LC3-GFP (Addgene, 210730), ubiquitin-GFP (Addgene, 11928), CFP (Addgene, 1179), GFP (Addgene, 1150), mtGFP - generous gift from Prof Rizzuto, mtRFP (Evrogen, FP147), dsRed (Addgene,
11151). Stable TSPO knockdown was achieved using the pGIPZ shRNA vector: clone ID V3LHS_331646, target sequence: 5'-TGAGTGTGGTCGTGAAGGC-3, purchased from Open Biosystems (Huntsville, AL, USA). Non-silencing shRNA vector sequences (mature antisense): V3LHS_331648: 5'-ACGCAGTAGTTGAGTGTGG-3' V3 LHS_331650: 5'-TCTGCAGGCCGGCGTACCA-3'. After transfection, cells were maintained for 2 weeks in media supplemented with $3 \mu \mathrm{g} / \mathrm{ml}$ puromycin (SERVA Electrophoresis $\mathrm{GmbH}$ ) to select transfected (GFP-positive) cells. TSPO KO SH-SY5Y cells were generated by transient transfection with GeneArt ${ }^{\mathrm{TM}}$ CRISPR Nuclease Vector with OFP Reporter Kit (Thermo Fisher, A21174), using a gRNA (5'-GGGCACGCTCTACTCAGCCA-3') targeting exon 2. Fibroblasts were obtained from Coriell Cell Repository (coriell.org) via MTA with HM; patient samples and Control samples were age-and sex-matched (details given in Table 1). Fibroblasts were cultured and maintained in EMEM (Gibco) as previously described [59]. Fibroblasts were passage matched for experiments and used between passages 10 and 15 .

\section{Immunocytochemistry}

Cells were initially plated on $22 \mathrm{~mm}$ glass coverslips at a $30 \%$ confluency and collected $48-72 \mathrm{~h}$ after transfection. At treatment completion, cells were washed once with $0.01 \mathrm{M}$ Phosphate Buffered Saline (PBS 1X) and fixed with 4\% paraformaldehyde (PFA) with a 10 min incubation. Cells were then washed three times for 5 min each with PBS 1X and permeabilised with a $0.5 \%$ Triton-X100/PBS $1 \mathrm{X}$ solution for 10-20 min. After another round of washes, cells were blocked for $1 \mathrm{~h}$ in a solution of PBS $1 \mathrm{X}$ containing $10 \% \mathrm{v} / \mathrm{v}$ goat serum (Thermo Fisher, 16210064) and 3\% w/v BSA. Primary antibody was incubated overnight at $4{ }^{\circ} \mathrm{C}$ in blocking solution at the following concentrations: 1:250 ATP5B (Abcam, ab14730), 1:250, TFEB (Cambridge

Table 1 The table details the information on the participant samples used for this study.

\begin{tabular}{lllll}
\hline Code & Status & Mutation & $\begin{array}{l}\text { Age at } \\
\text { biopsy (yrs) }\end{array}$ & Sex \\
\hline GM02189 & Control & & 63 & M \\
GM029510 & Control & & 55 & F \\
GM09400 & Control & & 61 & F \\
GM07924 & Control & & 52 & M \\
ND31618 & Parkin mutant & ARG42PRO & 63 & F \\
ND30171 & Parkin mutant & ARG42PRO/ & 54 & M \\
& & EX3DEL & & \\
ND40078 & Parkin mutant & ARG275TRP/ & 51 & F \\
& & ARG275GLN & & \\
ND37732 & Parkin mutant & EX3 40 BP DEL/ & 63 & F \\
& & EX4DEL & & \\
\hline
\end{tabular}


Bioscience, A303-673A) or 1:100 Tau (Abcam, ab32057). After three washes, the secondary antibody was incubated for $1 \mathrm{~h}$ at a 1:500 dilution of the following secondary antibodies in blocking solution: anti-mouse goat IgG Alexa 488 (Abcam, A11001), anti-rabbit donkey IgG Alexa 568 (Abcam, A10042), anti-mouse goat IgG Alexa 633 (Abcam, $\mathrm{A} 21050$ ). After $1 \mathrm{~h}$ at room temperature away from light, the coverslips were washed and mounted onto glass slides using a 4',6'-diamidino-2-phenylindole (DAPI)-containing mounting medium (ab104139). The coverslips were then imaged on a Leica SP5 confocal microscope.

\section{Western blotting}

Lysates were either made from mitochondrial fractions and whole cells. Fractions were collected in sucrose buffer (50 mM sucrose, $10 \mathrm{mMKCl}, 20 \mathrm{mM}$ HEPES, $1.5 \mathrm{mM}$ $\mathrm{MgCl} 2,1 \mathrm{mM}$ EDTA, $1 \mathrm{mM}$ EGTA [Sigma, E8145], protease inhibitor [Roche, 4693132001], $\mathrm{pH}$ 7.4) on ice. To break the plasma membrane, the cell suspension was passed through a 26-gauge needle (Sigma, Z192392) 30 times. Separation of the mitochondrial and cytoplasmic fractions was performed using differential centrifugation, as shown previously [60]. Primary antibodies: ACTB (Abcam, ab8826) 1:5000, ATP5B (Abcam, ab14730) 1:5000, ERK1/ 2 (Cell signalling, 9102S) 1:1000, pERK1/2 (Y202/Y204) (Cell signalling, 9101) 1:500, SDHA (Abcam, ab109865), TSPO (Abcam, ab109497) 1:4000, USP30 (Enzo, MBLPW0975) 1:1000, VDAC1 (Abcam, ab14734) 1:2000, 1:10000 Vinculin (Abcam, ab129002) and 1:1000 TFEB (Cambridge Bioscience, A303-673A). Imaging was carried using an ECL based method (GE, RPN2133) on a a BioRad ChemiDoc MP Imaging System, following incubation for $1 \mathrm{~h}$ with either anti-rabbit conjugated HRP 1:4000 (Dako, P0447), or rabbit anti-mouse conjugated HRP (Dako, P0448), or goat anti-rat IgG conjugated HRP 1:4000 (Abcam, ab97057). Densitometric analysis was performed using the ImageJ software. Values were normalised to ACTB loading control for whole-cell lysates and ATP5B for mitochondrial fractions.

\section{Immunohistochemistry}

Following PBS washing, slices were incubated at $80^{\circ} \mathrm{C}$ for $30 \mathrm{~min}$ in citrate buffer: $10 \mathrm{mM}$ sodium citrate dehydrate (Sigma, W30200), 0.05\% Tween-20 (Sigma, P7949), pH 6.0. Washes were followingly carried out in PBS-T $(0.05 \%$ Tween20 in PBS). Permeabilization was carried out for $15 \mathrm{~min}$ at $4{ }^{\circ} \mathrm{C}$ in $20 \mathrm{mM}$ HEPES, $300 \mathrm{mM}$ sucrose (Sigma, 59378), $50 \mathrm{mM}$ $\mathrm{NaCl}, 0.05 \%$ Triton-X100 (Sigma, T8787), $3 \mathrm{mM} \mathrm{MgCl}$, $0.05 \%$ sodium azide (Sigma, 71290 ). Blocking $-1 \mathrm{~h}$ at room temperature in 10\% horse serum (Thermo Fisher, 16210064), $1 \% \mathrm{w} / \mathrm{v}$ bovine serum albumin (BSA) (Sigma, A2153) in PBS
1X. Primary antibody incubation was carried out overnight at $4{ }^{\circ} \mathrm{C}$ first using a $1: 150$ dilution of rabbit TSPO antibody (biorbyt, orb5845) and the next day, after washing, using rat DAT antibody (Millipore, MAB369), 1:150 in blocking solution. Secondary antibodies: anti-rat goat IgG Alexa 555conjugated (abcam, ab105158), anti-rabbit goat IgG Alexa 488-conjugated (Invitrogen, A11008) at 1:200 in blocking solution for an hour at RT, while shaking. Slices were washed 4-5 times in PBS-T $1 \mathrm{X}$ and three times in PBS $1 \mathrm{X}$, before a 5 min incubation with $600 \mathrm{nM}$ DAPI solution (D1306, Invitrogen). Excess DAPI was cleared with three 5 min washes in PBS 1X and slices were then mounted on glass slides (VWR, 631-0908) using a polyvinyl alcohol mounting medium containing 1,4-diazabicyclo[2.2.2] octane $\left(\mathrm{DABCO}^{\circledR}\right)$ (Sigma, 10981), covered with $22 \mathrm{~mm}$ glass coverslips. The slices were then imaged on a confocal Leica SP5 with a 63x lens. TSPO intensity was gathered from DAT stained projections within the globus pallidus and entopeduncular nucleus of multiple stained slices $(n=3)$. Equal numbers of neurons from each slice and each area were compared.

\section{Quantitative PCR}

Quantitative reverse-transcription PCR (qRT-PCR) was carried out with a standard calibration curve containing serial dilutions of each cDNA transcript for each separate gene. The mRNA isolation step was carried out using the RNeasy Plus Mini kit (Qiagen, 74134), as per manufacturer's instructions. cDNA synthesis was carried out using the QuantiNova ${ }^{\mathrm{TM}}$ Reverse Transcription Kit (Qiagen, 205411), in an RNase-free environment. Only RNA samples with $260 / 280 \mathrm{~nm}$ of $2.0 \pm 0.2$ were used. Real-time PCR, executed with the QuantiNova SYBR Green PCR kit (Qiagen, 208052), was used to amplify the standards and then quantify the sample's mRNA expression. Using 96well PCR plates (Thermo Fisher, AB0800W), covered by adhesive seals (4titutde, 4ti-0565), triplicates of each sample were mixed with SYBR green dye and appropriate primers. Details: MAOB (proprietary, qHsaCID0017743), TSPO (proprietary, qHsaCED0037846), USP30 (S: GGG ATTATAGACCGGACACTA, AS: CACAAGCCCTTTT CTACGCT); 18S (S: CGCGGTTCTATTTTGTTGGT, AS: AGTCGGCATCGTTATGGTC). The 96-well plate was placed into a CFX Connect ${ }^{\mathrm{TM}}$ Real-Time PCR Detection System, and 40 cycles of denaturation $\left(5 \mathrm{~s}, 95^{\circ} \mathrm{C}\right)$ and extension $\left(10 \mathrm{~s}, 60{ }^{\circ} \mathrm{C}\right)$ were executed.

\section{Redox analysis}

Dynamic analysis of cellular redox was carried out using four different live cell imaging dyes, that detect specific molecular species: cytoplasmic superoxide is analysed using DHE (Life Technologies, D11347); mitochondrial 
superoxide is detected with mitoSOX ${ }^{\mathrm{TM}}$ Red (Life Technologies, M36008); global cellular hydrogen peroxide $\left(\mathrm{H}_{2} \mathrm{O}_{2}\right)$ and peroxyl radicals $\left(\mathrm{HO}_{2}\right)$ are measured using DCFDA (Sigma, D6883) while unbound reduced glutathione (GSH) is assayed with MCB (Life Technologies, M-1381MP). Cells were cells co-transfected with an appropriate fluorophore that would not interfere with the fluorescence of the dye. All dyes were administered to the recording medium (RM) onto cells plated on coverslips, within Attafluor ${ }^{\circledast}$ metal cell chambers (Molecular Probes ${ }^{\mathrm{TM}}$, Thermo fisher, A-7816). RM composition is: $5.6 \mathrm{mMKCl}$ (Sigma, P9333), $10 \mathrm{mM} \mathrm{D-(+)-Glucose} \mathrm{(Sigma,} \mathrm{G7528),}$ $10 \mathrm{mM}$ HEPES (Sigma, H4304), $4.2 \mathrm{mM} \mathrm{NaHCO}{ }_{3}$ (Sigma, 56297), $138 \mathrm{mM} \mathrm{NaCl}$ (Sigma, S5886), $2.6 \mathrm{mM} \mathrm{CaCl} 2$ (Sigma, C7902), $1.2 \mathrm{mM} \mathrm{NaH} \mathrm{PO}_{4} \quad(\mathrm{BDH}, 1024940)$, $1.2 \mathrm{mM} \mathrm{MgCl}$ (BDH, 10149), $\mathrm{pH}$ 7.4.

DHE $(10 \mu \mathrm{M})$, mitoSOX $(10 \mu \mathrm{M})$, and DCFDA $(15 \mu \mathrm{M})$ were diluted in RM and imaged onto a Zeiss LSM510 confocal microscope. MCB was used at final concentration of $2.5 \mu \mathrm{M}$ in RM. Brightness controlling settings were maintained consistently within the experiment for all techniques.

\section{mtKeima measurement}

To analyse the delivery of mitochondrial content to the lysosome, we used a plasmid vector expressing mt-Keima ([Amalgaam, AM-V0251]). Cells were transfected with mtKeima, treated, and successively imaged on a Leica SP5 microscope while in $\mathrm{RM}$ buffer at $37^{\circ} \mathrm{C}$. Using ImageJ, ratio pictures of $586 / 440 \mathrm{~nm}$ excitation were created, and the number of pixels emitting only red signal over the total number of pixels per cell was used as mitophagy index.

\section{Imaging of mitochondria}

To image the lysosomal population, cells were transfected with DsRed and TSPO siRNA/nsc, treated in culture media, stained with $100 \mathrm{nM}$ LysoTracker ${ }^{\mathrm{TM}}$ DND-22 (Thermo Fisher, L7525) for $30 \mathrm{~min}$ and then imaged on a Leica SP5, in RM. Their number or volume was quantified on ImageJ, using the "3D object counter" plugin. Mitochondrial morphology was assayed using cells transfected with a mitochondria-targeted GFP (mtGFP), utilising only cells with neurites. Aspect Ratio (AR), a measure of elongation, and Form Factor (FF), the inverse of circularity and a measure of branching, were calculated on the whole mitochondrial network, with ImageJ software. $\Delta \Psi_{\mathrm{m}}$ was measured using tetramethylrhodamine methyl ester (TMRM). Fluorescence intensity was directly measured after incubation of $50 \mathrm{nM}$ TMRM in RM for $30 \mathrm{~min}$ on a Zeiss LSM510 confocal microscope. To measure basal membrane potential, only mitochondrial ROIs were used, while the rate of $\left.\mathrm{MPP}^{+}\right)$-induced depolarisation was measured using live recordings of whole-cell ROIs.

\section{Transcriptome data analysis}

Using the CRISPR/Cas9 system, we generated a line of SHY-SY5 ablated for TSPO gene (SHY-TSPO KO). This and the control line were exposed to $\mathrm{MPP}+$ before extracting the total RNA. Total RNA samples from TSPO WT and KO cells were extracted using TRIzol reagent (Invitrogen; 374 Thermo Fisher Scientific, Waltham, MA, USA) and then quantified with an Agilent 2100 bioanalyzer. The quantified RNA samples were used for NEBNext ${ }^{\circledR}$ rRNA-depleted (Human/Mouse/Rat) stranded library preparation. Library preparation and RNA sequencing was conducted at the UCL Genomics. Libraries were prepared using non-strand specific Illumina TruSeq Sample Preparation Kits followed by Illumina sequencing. FASTQ files were aligned using TopHat and Cufflinks. Normalisation and differential analyses were carried out using $\mathrm{R}$ software Bioconductor [61] package DESeq2 [62], and gene set enrichment analysis (GSEA) was carried out using EGSEA [63].

\section{Assessment of cell viability}

Cell viability was measured using propidium iodide (PI, Sigma, P2667-2OTST). Cells were co-transfected with GFP in six well plates. After treatment, wells were washed with PBS once and successively incubated for 10 min with $3 \mu \mathrm{g} /$ $\mathrm{ml}$ of PI in RM. Three washes with PBS were used to remove excess stain. The cells were then maintained in RM and imaged immediately using the Leica DMIRB inverted fluorescence microscope. The proportion of PI-positive cells within the transfected population was used as a measure of viability. The trypan blue exclusion method $(100,300 \mu \mathrm{M}$, Sigma) was also enrolled, and cells counted under the KOVA $^{\circledast}$ Glasstic Slide with Counting Grids.

\section{Data analysis}

Data are presented as mean \pm standard error of the mean. Statistical analysis was performed using R software (version 3.0.2). All analyses were carried out in a standardised manner. In summary, to determine whether the sample distributions were normal, a Shapiro-Wilk test was performed for sample groups with $N<50$; for sample groups with $N \geq 50$, a histogram and a quantile-quantile were employed. For experiments with two normally distributed conditions, a Welch's $t$-test was performed. If the experiment included multiple conditions, a one-way analysis of variance (ANOVA) tests was performed to address if the variance between samples was smaller than between conditions, (i.e. vehicle-treated \pm TSPO cells to MPP + treated). Comparisons between conditions were carried out using a pairwise $t$-test, utilising Holm's correction. If any sample 
failed the normality distribution tests, a Wilcox test was used to compare two conditions, a Kruskal-Wallis test was used to compare groups of conditions and a Wilcoxon rank sum tests with Holm's multiple correction for pairwise comparisons. The $\mathrm{N}$ numbers utilised indicate the number of biological repeats. In assays that compare bulk groups of cells, i.e. WB or cell viability, 3 or 4 separate sets of experiments (and treatments) were compared, with technical repeats (multiple measurements of the same biological sample) averaged out. In imaging experiments, two separate treatments and transfections were carried out, and the number of cells analysed per condition is reported as N. In the PET and autoradiography experiments, the $\mathrm{N}$ number indicates the total number of animals used per condition. Statistical significance is indicated as follows: ' $*$ ' $-p<0.05$; '**' $-p<0.01$; '***' $-p<0.001$.

\section{Results}

\section{TSPO expression increases in Parkinson's disease}

Our analysis began by assaying the accumulation of TSPO in vivo using second-generation ligands for the protein in a PD rat model. Animal brains were scanned via PET 9 days after unilateral injection of 6-OHDA. Based on the data collected, a heat map representing the binding of the radiolabelled, high-affinity [16, 29] TSPO ligand $\left[{ }^{11} \mathrm{C}\right]$ PBR28 was generated (Fig. 1A). This effect was quantified via biodistribution analysis (Fig. 1B), indicating a marked upregulation of $\left[{ }^{11} \mathrm{C}\right] \mathrm{PBR} 28$ uptake in the brains of 6OHDA-injected animals when compared to the shaminjected group. Moreover, the extent of $\left[{ }^{11} \mathrm{C}\right] \mathrm{PBR} 28$ uptake after 6-OHDA injection was significantly higher in the ipsilateral brain hemisphere of treated animals than in the contralateral one. Further analysis of coronal sections of the central nervous system (CNS) of rats subjected to the same treatment was run via autoradiography, using radiolabelled PBR28 (Fig. 1C). The quantification in 1D showed a generalised increase in radioactive signal in the ipsilateral hemisphere of 6-OHDA-injected animals, confirming the PET's trend observed previously. Even though the accumulation of TSPO is commonly acknowledged to be occurring in glial and microglial cells, neurons can express the protein too (Fig. 1E) [64]. In order to test if dopaminergic neurons can upregulate TSPO following treatment with neurotoxins, we carried out an immunohistological analysis of coronal brain sections of 6-OHDAtreated rats $(n=3)$. Different midbrain sections were selected, labelled with the nuclear stain 4',6-diamidino-2phenylindole, dihydrochloride (DAPI), and an immunoassay was run for TSPO and dopamine transporter (DAT) [65-67] (Fig. 1F, G). By looking at the globus pallidus and the entopeduncular nucleus, we found that dopaminergic neurons revealed a predominant co-localisation between TSPO and DAT-positive, and following treatments with the neurotoxins, TSPO was markedly overexpressed (Fig. 1F, G). Validation of TSPO upregulation in PD rat models and its neurotoxin-induced accumulation in dopaminergic neurons represented the basis for the next in vitro set of analyses, which began by exposing SH-SY5Y cells to 6-OHDA $(50 \mu \mathrm{M}, 4-8 \mathrm{~h})$ and measuring TSPO levels via Western blotting (WB) (Fig. 1H, I). The analysis corroborated the results obtained both in vivo (Fig. 1A, B) and ex vivo (Fig. 1F, G). To further the relationship between PD-related mutations and increased TSPO expression, fibroblasts from PD patients $(n=4)$ bearing point mutations and/or exon deletions on the PARK2 gene (Table 1) were characterised by significant TSPO over-expression when compared to age-and-sex-matched controls. (Fig. 1J-L).

\section{TSPO upregulation is the result of an ERK1/2- dependent transcriptional response}

Having observed that SH-SY5Y cells recapitulate the upregulation of TSPO observed in vivo, we administered two other PD-linked neurotoxins, chosen for their ability to induce ERK-dependent autophagy and Parkinsonism in vivo [68-71] (Table 2). By administering rotenone (Rot) $(100 \mathrm{nM})$, we monitored the level of TSPO over time $(4,6$, and $8 \mathrm{~h}$ ) (Fig. 2A, B). The results showed that the increase in the expression of TSPO was achieved already at $4 \mathrm{~h}$ of treatment, and there were no significant differences between the time points tested (Fig. 2A, B). The second neurotoxin used, MPP + , had shown a more pronounced increase, compared to Rot, in TSPO previously [72-75], at a concentration of $0,5 \mathrm{mM}$ in $8 \mathrm{~h}$; therefore, we focused our subsequent analyses on this neurotoxin. The activation of ERK1/2 induces TSPO transcription via activator protein 1 (AP1) and signal transducer and activator of transcription 3 (STAT3).

To assess whether MPP+induced upregulation of TSPO expression was dependent on ERK1/2, we treated the cells with U0126 $(5 \mu \mathrm{M}, 8 \mathrm{~h})$, a pharmacological inhibitor of mitogenactivated protein kinase (MEK)-induced ERK1/2 phosphorylation. As shown in Fig. 2C, D, the co-administration of U0126 prevented the upregulation of TSPO in response to $\mathrm{MPP}+$. At confirmation of the MPP + dependent increase of $T S P O$ transcription, mRNA levels were assessed via qRT-PCR (Fig. 2E). SH-SY5Y cells were treated for a longer time (20 h), and the results showed a significantly higher expression of TSPO mRNA levels than untreated cells (Fig. 2E). Then we tested the effect of MPP $+(0.5 \mathrm{mM})$ at 8 and $20 \mathrm{~h}$, looking at different mitochondrial proteins (Fig. 2F-H). Remarkably, the inner mitochondrial membrane proteins ATP synthase (ATP5B) and succinate dehydrogenase complex (SDHA) 
A

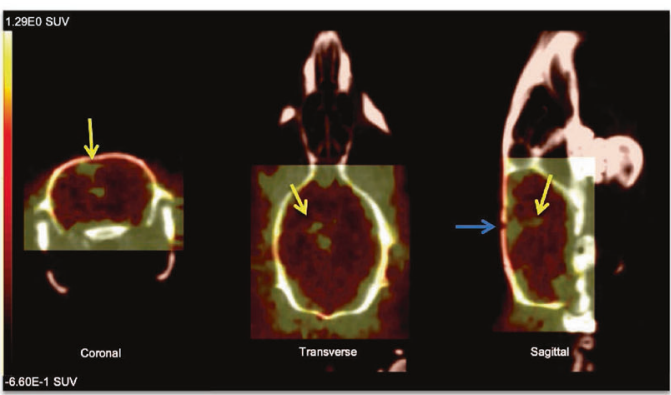

B

Uptake of $\left[{ }^{11} \mathrm{C}\right] \mathrm{PBR} 28$ in the brain of Sprague-Dawley rats with or without 6OHDA lesions

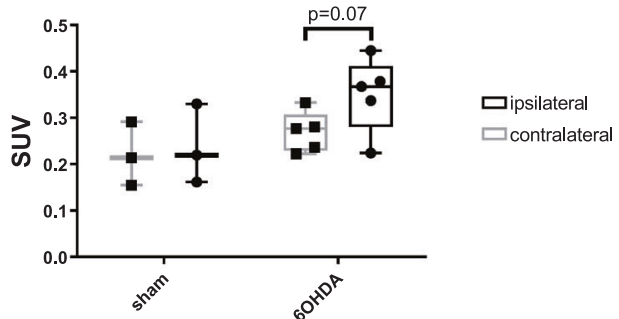

C

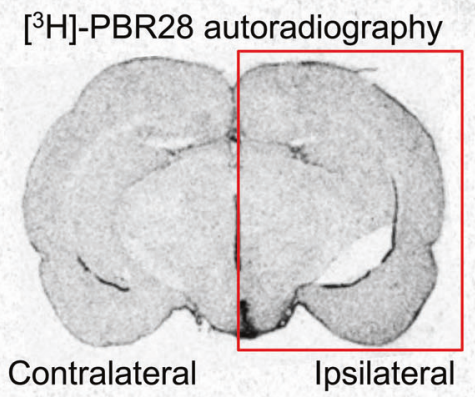

$\mathbf{F}$

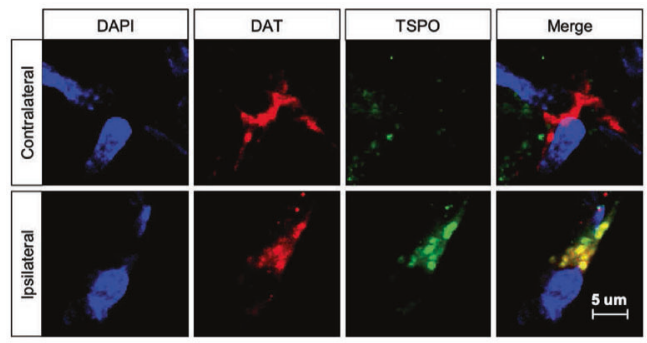

D

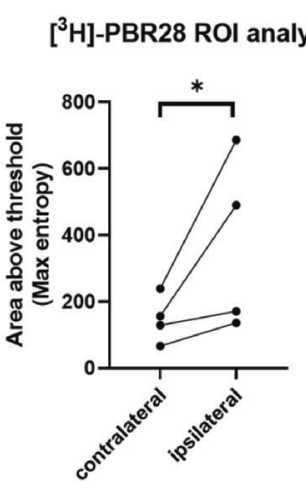

G

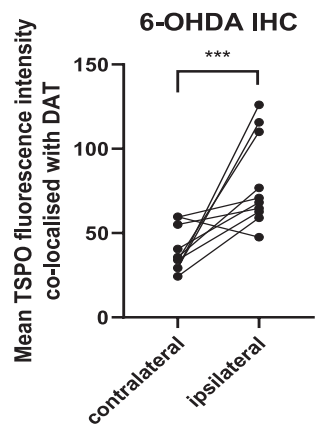

E

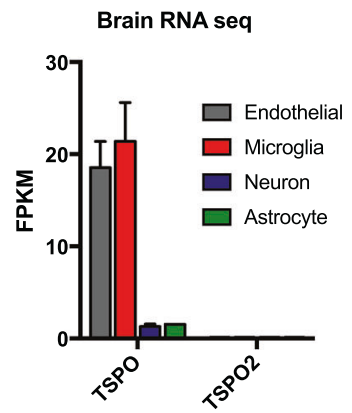

H

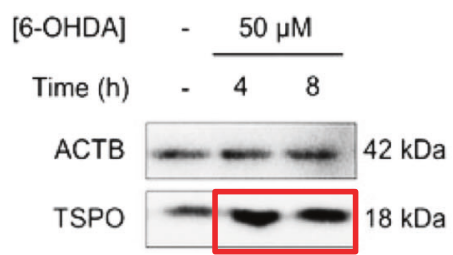

I

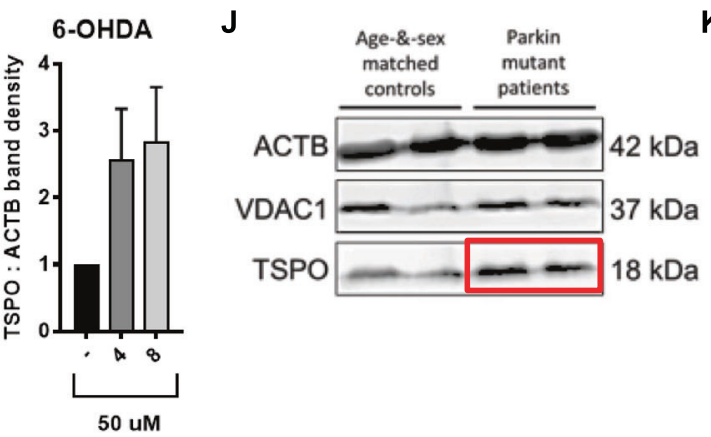

K

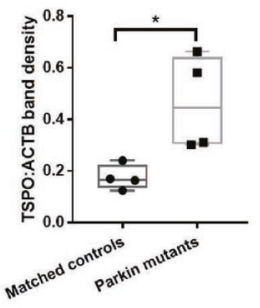

$\mathbf{L}$

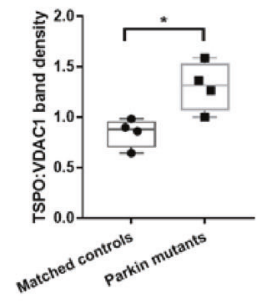

remained substantially unchanged (Fig. 2F-H). Perhaps unsurprisingly, the OMM protein voltage-dependent anion channel (VDAC1), to which TSPO is molecularly and functionally linked [60, 76, 77], increases its expression after $20 \mathrm{~h}$ of treatment by an average of 20\% (Supplementary Fig. 1A-D). The hypothesis whereby the accumulation of
TSPO represents a pro-pathological mechanism was corroborated further by the degree of cell death in SH-SY5Y in response to MPP+ using dosages (Fig. 2J, K) equally able to collapse the mitochondrial membrane potential $\left(\Delta \Psi_{\mathrm{m}}\right)$ (Supplementary Fig. 2A-F). A low but sustained dose of the MPP+ damaged cells expressing TSPO (Control and +TSPO), 
Fig. 1 In vivo and in vitro upregulation of TSPO by Parkinsonian toxins. A A representative PET image of $\left[{ }^{11} \mathrm{C}\right] \mathrm{PBR} 28$ uptake in the brain of a Sprague-Dawley rat subjected to a unilateral injection of 6OHDA for 9 days. Yellow arrows indicate representative areas of high uptake; the stereotaxic drill hole is indicated in the sagittal view by a blue arrow. B Bar charts displaying the average $\left[{ }^{11} \mathrm{C}\right] \mathrm{PBR} 28$ uptake in the whole hemisphere of 6-OHDA lesioned rats $(n=5)$, with contralateral control hemisphere, and of sham samples $(n=3)$, subjected to an injection of saline. $\mathbf{C}$ Representative autoradiography section of unilateral 6-OHDA lesioned rats displaying $\left[{ }^{3} \mathrm{H}\right]-\mathrm{PBR} 28$ uptake, with a (D) threshold-based image quantification of $\left[{ }^{3} \mathrm{H}\right]-\mathrm{PBR} 28$ signal comparing whole ipsilateral to contralateral hemispheres. E Comparison of TSPO expression in endothelial, microglia, neuron, and astrocyte tissues using a public mRNA database [64]. F Representative immunohistological images of coronal sections (contralateral and ipsilateral origin) of the 6-OHDA-treated rats stained for DAPI, TSPO, and DAT. G Image-based quantification of co-localised TSPO signal intensity in DAT-stained neurons from different midbrain sections ( $n=10$ cells per condition). H Representative bands of WB of TSPO and loading control ACTB in whole SH-SY5Y cell lysates, under the administration of $50 \mu \mathrm{M}$ 6-OHDA for 4 or $8 \mathrm{~h}$, with, in (I), its ACTBnormalised band density $(n=4)$. J Representative image from a SDSPAGE analysis of TSPO expression, controlled against ACTB and the mitochondrial VDAC1, in fibroblasts of Parkin mutant PD patients, with respective age-\&-sex-matched controls. Band density quantification of TSPO expression normalised $(\mathbf{K})$ the loading control ACTB and $(\mathbf{L})$ to the mitochondrial control VDAC1 $(n=4)$.

leaving unharmed those in which the protein was downregulated (-TSPO) (Fig. 2J). An opposite outcome was measured with the administration of MPP+ at a higher concentration, which was detrimental for neurons devoid of TSPO (Fig. 2K). Such an observation on cell death susceptibility was expanded further by using dopamine in toxic concentrations $(100,200$, and $300 \mu \mathrm{M})$ and confirmed in differentiated SHSY5Y cells (Fig. 2L).

\section{TSPO shifts the cellular redox balance of neurons}

Subsequently, we adopted live-cell imaging tools to monitor different subcellular pools of ROS in SH-SY5Y cells mocktransfected (Control), transiently over-expressing TSPO (+TSPO) or down-regulated for the protein (-TSPO). We initially administered MPP + in acute exposure $(4 \mathrm{mM}$, $15 \mathrm{~min}$ ) to cells loaded with dihydroethidium (DHE), which allows measuring the cytoplasmic pools of superoxide. Figure $3 \mathrm{~A}-\mathrm{C}$ showed that, at steady state, cytoplasmic $\mathrm{O}_{2}{ }^{\bullet}$ is heightened solely in +TSPO cells, with levels that are comparable to control cells after MPP + treatment. While control cells underwent an increase in signal following MPP + administration, - TSPO cells did not show any relevant change. By using the mitochondrially targeted, superoxide indicator MitoSOX ${ }^{\mathrm{TM}}$ (mitoSOX) as a read-out of mitochondrial ROS (mROS) (Fig. 3D, E), we instead observed that basal mitoSOX fluorescence emission was almost doubled in -TSPO cells when compared to control conditions, and that acute treatment with $\mathrm{MPP}+(4 \mathrm{mM}$, $15 \mathrm{~min}$ ) lowered its intensity. Oppositely, the over- expression of TSPO (+TSPO) counteracted the MPP+ increase in mitoSOX fluorescence (Fig. 3D, E). Analysis of global cellular hydroxyl radicals using $2^{\prime}, 7^{\prime}$-Dichlorofluorescin Diacetate (DCFDA) fluorescence showed the same trend observed with DHE (Fig. 3F, G). And thus, -TSPO cells display significantly lower accumulation of DCFDA fluorescence than Control or +TSPO cells and are protected from MPP+induced increase in ROS levels.

Control cells showed instead a statistically significant increase in global cellular hydroxyl radicals after acute treatment with MPP + which is ineffective on +TSPO cells which feature a more oxidised basal redox balance at resting conditions. To monitor the effect of TSPO modulation on the antioxidant capacity of SH-SY5Y cells, we then analysed reduced GSH levels via monochlorobimane (MCB). The results showed that + TSPO cells are highly sensitive to the MPP+-induced generation of ROS, which causes a significant drop in the pool of reduced GSH that was not observed in either Control or -TSPO cells (Supplementary Fig. 1E-G). To further address the opposite relationship between cytoplasmic and mROS pools, we used superoxide scavengers to remove, selectively, ROS from each intracellular compartment. By employing the artificial antioxidant TEMPO or its mitochondrially targeted version: mitoTEMPO, we measured the compartment-specific pools of oxidised GSH, interpolated from the increase in MCB signal following scavenger treatment (Supplementary Fig. 1H). This revealed that -TSPO cells had oxidised GSH in both compartments, while + TSPO cells only in the mitochondria. In addition, mitoTEMPO does not shield + TSPO cells, like TEMPO, we infer that these cells are very susceptible to MPP+induced ROS in the cytoplasm.

Knowing that TSPO engages the $\mathrm{Ca}^{2+}$-dependent NADPH oxidases (NOX) [60, 78] to generate ROS, we tested whether monoamine oxidases (MAOs) [79-81] were involved in this redox rewiring. A qRT-PCR analysis revealed that + TSPO cells present vastly increased levels of MAOB (Fig. 3H). Finally, the pro-oxidant phenotype induced by TSPO over-expression is paired with a disorganised and dense mitochondrial network, which is more elongated and branched in -TSPO cells. However, TSPO depletion predisposes mitochondria to undergo a drastically more severe fragmentation after exposure to $\mathrm{MPP}+$ (Fig. 3J, K). Therefore, TSPO over-expression induces an imbalance in redox production, which is likely to promote toxicity by neurotoxins leading to the assessment of mitophagy response in these conditions.

\section{The upregulation of TSPO de-ubiquitylates mitochondria}

Mitophagy can function as a quality-control stress response to remove damaged mitochondria. It can be induced by a variety 
Table 2 The table shows the three Parkinsonian neurotoxins used in the study, their chemical structures and their neurotoxic properties.

\begin{tabular}{llll}
\hline Acronym & Name & 6-hydroxydopamine \\
6-OHDA & rotenone & It is an hydroxylated analogue of dopamine \\
rotenone & 1-methyl-4-phenylpyridinium & $\begin{array}{l}\text { Inhibits the transfer of electrons from iron-sulfur } \\
\text { centers in complex I to ubiquinone }\end{array}$ \\
Interferes with the oxidative phosphorylation in \\
mitochondria by inhibiting complex I
\end{tabular}

of toxic or non-toxic triggers [82] and be carried out by different pathways [83]. Within the context of neurodegeneration, the PINK1-Parkin pathway has been linked more strongly to pathology and studied at highest depth [7]. However, previous studies showed an activation of Parkinindependent mitophagy following neurotoxin addition [70, 71, 84, 85]. Therefore, we monitored the steps of the mitophagic cascade in response to MPP + . We began by enrolling the mtKeima reporter, which measures mitochondrial delivery to lysosomes, comparing Control, +TSPO, and -TSPO SH-SY5Y cells (Fig. 4A). TSPO downregulation induced a significant increase in mitophagy index both at resting conditions and after MPP+ treatment, while TSPO overexpression induced the opposite but less pronounced phenomenon. Oppositely, the mitochondrial uncoupler carbonyl cyanide-4-(trifluoromethoxy) phenylhydrazone (FCCP), commonly used to activate PINK1-Parkin-mediated mitophagy, induced a robust and comparable increase in mitophagy index in Control and -TSPO cells, but a strong reduction caused by TSPO overexpression. Notably, the MPP +induced increase in signal was quantified as being $30-50 \%$ that of FCCP-treated cells (Fig. 4A).

To confirm the involvement of the autophagy pathway in response to MPP + treatment, we measured autophagosome and Parkin recruitment on mitochondria in SH-SY5Y cells expressing the fluorescent reporters LC3-GFP (Fig. 4B, C) and Parkin-YFP (Fig. 4D, E), calculated as a number of mitochondrial LC3 punctae, or mitophagosomes, per cell. Bafilomycin 1A (BAF) $(10 \mathrm{nM})$, an inhibitor of the vacuolartype $\mathrm{H}(+)$-ATPase, was used to block lysosomal acidification and prevent autophagic degradation [86], therefore allowing the accumulation of the mitophagosomes. Both analyses demonstrated that MPP + treatment does not block the translocation of Parkin on mitochondria. Therefore, in order to gain insights into the mechanism via which TSPO inhibits mitophagy during MPP + treatment, we assessed the levels of mitochondrial ubiquitination in Control and TSPO SH-SY5Y cells by measuring the degree of co-localisation between ATP5B and ubiquitin. Notably, repression of TSPO activity increased basal levels of mitochondrial ubiquitination (Supplementary Fig. 3I-L), which were increased further when BAF was combined with MPP+ (Fig. 4F, G). Oppositely, TSPO over-expression, simulating the upregulation seen after treatment, increased the level of the deubiquitinase (DUB) ubiquitin specific peptidase 30 (USP30), as shown both via WB (Fig. 4H, I) and a qRT-PCR analysis (Fig. 4J). USP30 is the sole mitochondrially localised DUB [87] that impairs basal [88] and Parkin-mediated mitophagy [46]. Its inhibition has been proven to induce basal mitophagy upstream of PINK1, without inducing Parkin translocation [88]. To confirm the engagement of USP30 in the mitophagy pathway inhibited by TSPO, we transfected PINK1 KO SH-SY5Y cells (Fig. 4K) with mtKeima and measured the number of mitochondria removed by lysosomes in the presence and absence of TSPO (Fig. 4L). As expected, we observed an abolishment of the pro-mitophagic effects of TSPO interference, alongside the absence of any effect induced by FCCP.

Nevertheless, MPP + generated a significant increase in the mitophagy index of control cells, indicating that, in the presence of TSPO, the neurotoxin induces mitochondrial delivery to lysosomes without engaging the PINK1-Parkin 
A

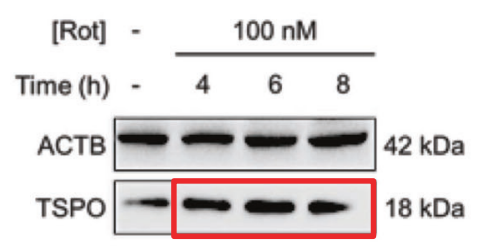

C

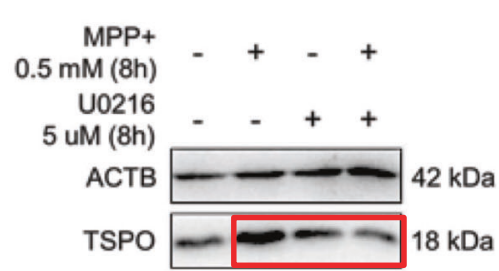

B

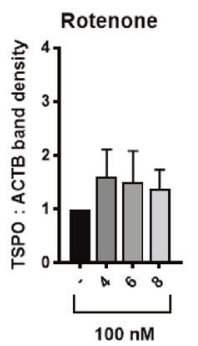

D

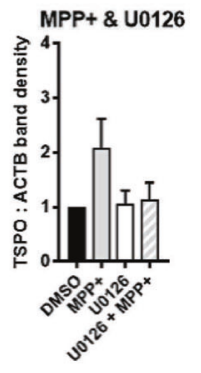

E

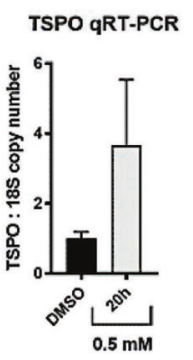

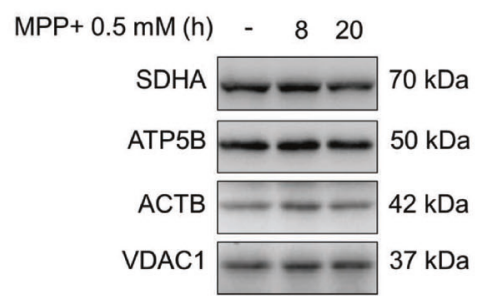

J

Sub-lethal $(0.5 \mathrm{mM} 20 \mathrm{~h})$

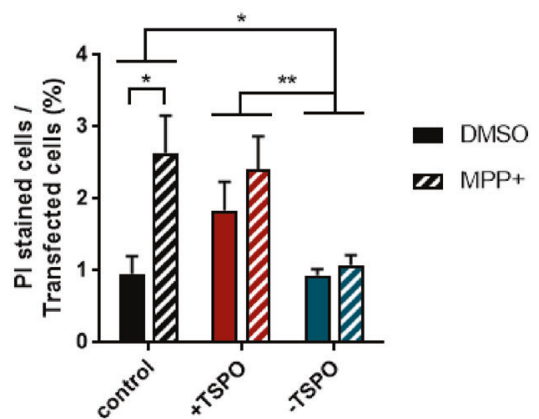

G

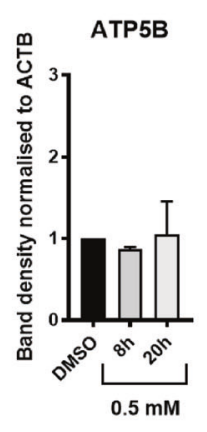

H

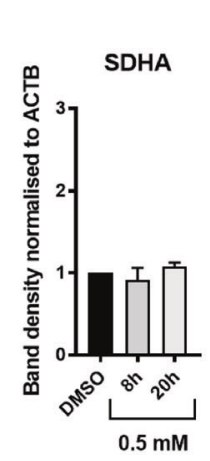

I

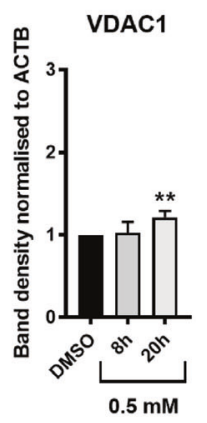

K

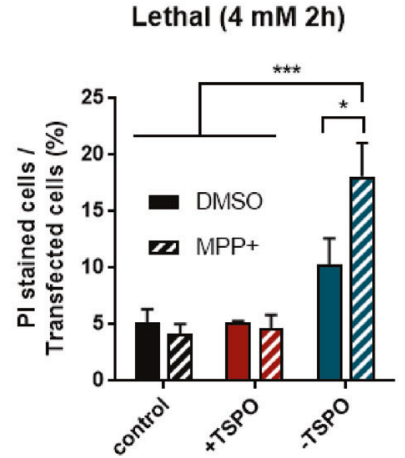

$\mathbf{L}$

Trypan blue

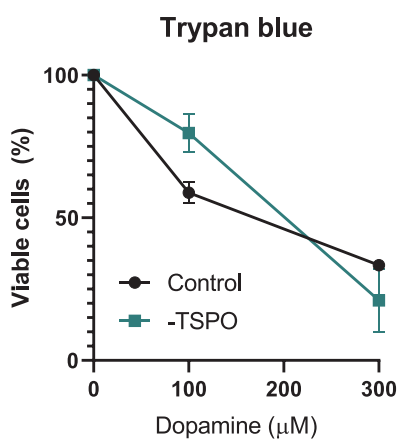

Fig. $2 \mathrm{MPP}+$ induces an ERK-dependent transcriptional upregulation of TSPO. A Representative WB bands of SH-SY5Y cells treated with $100 \mathrm{nM}$ Rot for 4,6 , and $8 \mathrm{~h}$. Whole-cell protein lysates were probed for TSPO and $\beta$ - actin (ACTB). B Average band densities were normalised to ACTB, $(n=3)$. C Representative WB bands of TSPO and ACTB in SH-SY5Y whole-cell lysates, following administration of $0.5 \mathrm{mM} \mathrm{MPP}+$ for $8 \mathrm{~h}$, in absence or presence of the MEK inhibitor U0126. TSPO band densities are given in (D) $(n \geq 6)$. E The mRNA levels of both TSPO and $18 S$ ribosomal RNA were measured by reverse transcription quantitative real-time PCR. TSPO absolute copy number was normalised over $18 \mathrm{~S}(n=3)$. F Representative SDS-
PAGE of wild type SH-SY5Y cells treated (Control) for 8 and $20 \mathrm{~h}$ with $0.5 \mathrm{mM}$ MPP + , immunoprobed for the IMM proteins ATP5B and SDHA, the OMM protein VDAC1 and the loading control ACTB. Band density quantification of the expression of $(\mathbf{G})$ ATP5B, (H) SDHA, (I) VDAC1 normalised to ACTB $(n \geq 3)$. J, K PI stained-totransfected cell ratio of cells treated with MPP + dosages $(0.5 \mathrm{mM}$ for $20 \mathrm{~h}, 4 \mathrm{mM}$ for $2 \mathrm{~h}$ ) and expressing different TSPO levels $(n=3)$. L Trypan blue assay of Control and - TSPO cells following challenge with various dopamine concentrations $(100,200$, and $300 \mu \mathrm{M})$. Results are presented as the ratio of living cells compared to non-treated. 
A

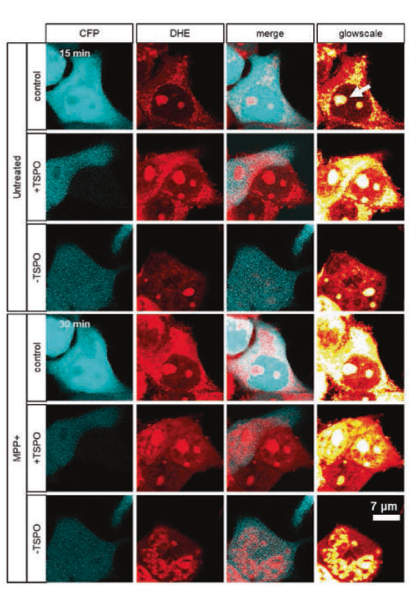

$\mathbf{F}$

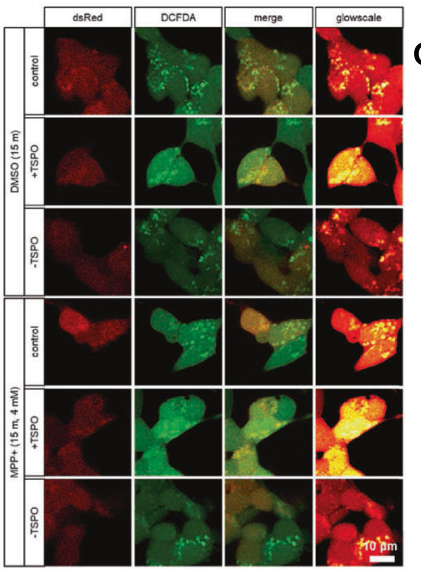

$J$

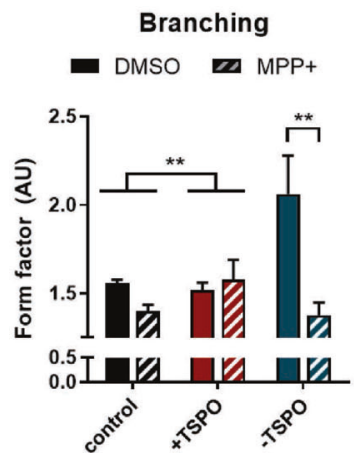

Fig. 3 TSPO drives cellular redox-stress response triggered by the neurotoxin MPP + . A Representative DHE analysis images in \pm TSPO cells transfected with CFP and treated with $4 \mathrm{mM} \mathrm{MPP}+$ in acute (15 min). B Representative fluorescent traces and (C) linear DHE increment quantification for cells transfected with TSPO and treated with MPP $+\left(n \geq 15\right.$ cells). D Representative images of mitoSOX ${ }^{\mathrm{TM}}$ staining of \pm TSPO cells co-transfected with CFP and treated with $4 \mathrm{mM}$ MPP + shortly (15 min) before imaging. E Quantification of mitochondrial mitoSOX intensity from (D) $(n \geq 20$ cells). F DCFDA analysis of cellular peroxyl radicals in cells transfected for TSPO and
D

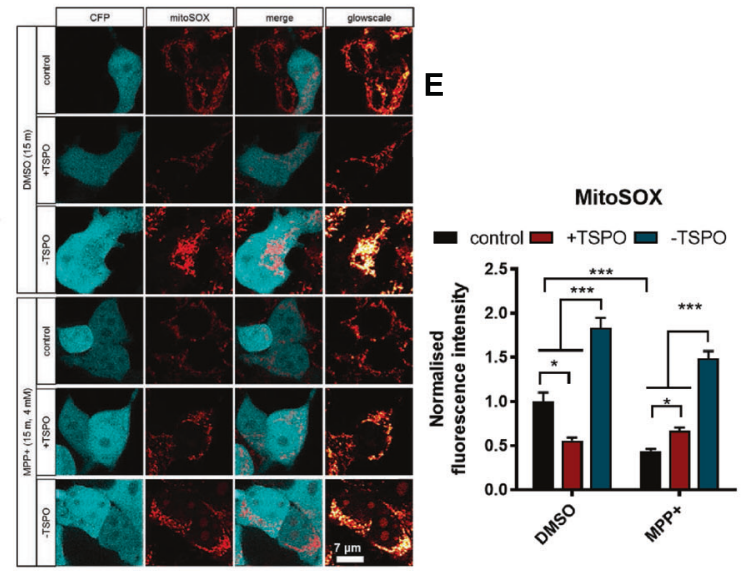

H

MAOB qRT-PCR
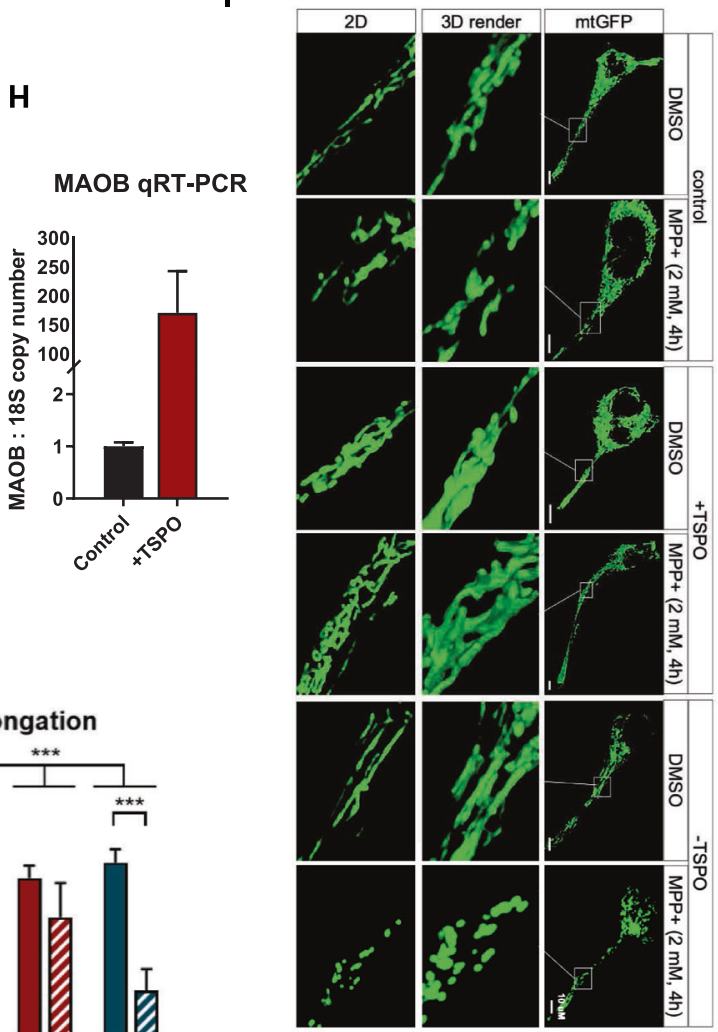
average DCFDA fluorescence count normalised to control $(n \geq 20$ cells). H qRT-PCR of Control and +TSPO cells with primers for human MAOB. Absolute copy numbers were quantified, normalised to the loading control, the $18 \mathrm{~S}$ gene, and normalised to transfection control. $(n=3)$. I Mitochondrial morphology analysis, using mtGFP, in \pm TSPO cells treated with MPP $+(2 \mathrm{mM})$ for $4 \mathrm{~h}$. Two mathematical image-based analyses were made: $(\mathbf{J})$ form factor, a measure of branching, and $(\mathbf{K})$ aspect ratio, a measure of elongation ( $n=10$ cells). 
A

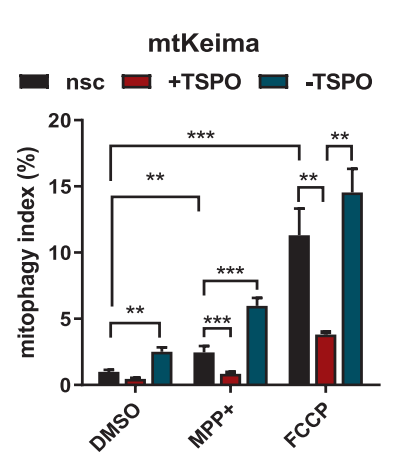

D

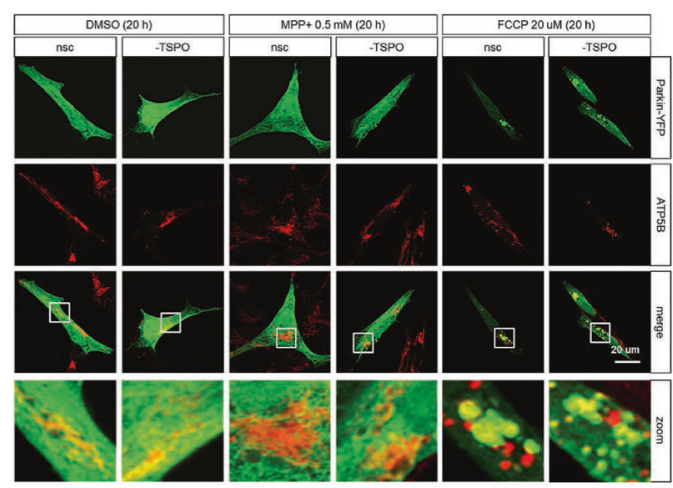

$\mathbf{F}$
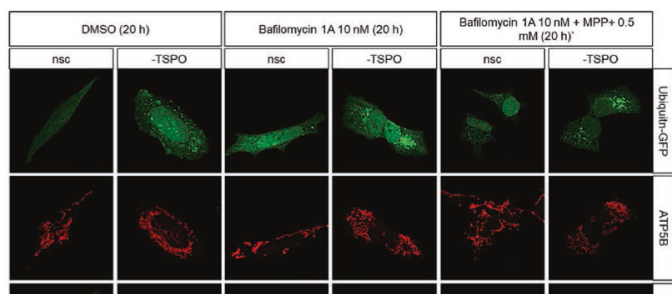

(1)

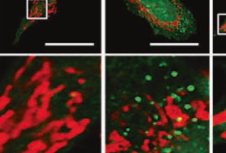

H

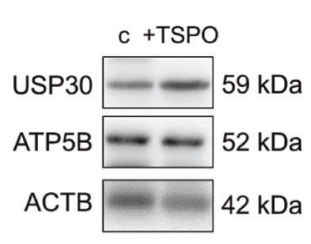

B

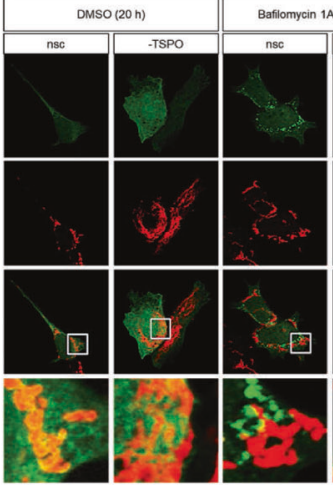

남
圆

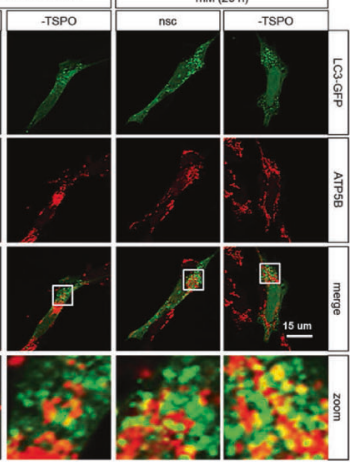

C

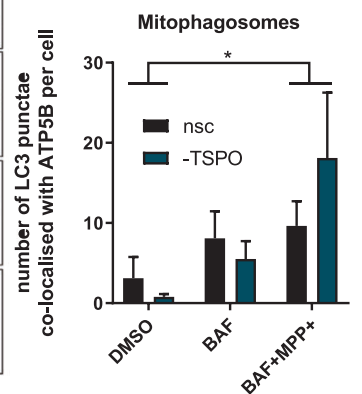

E

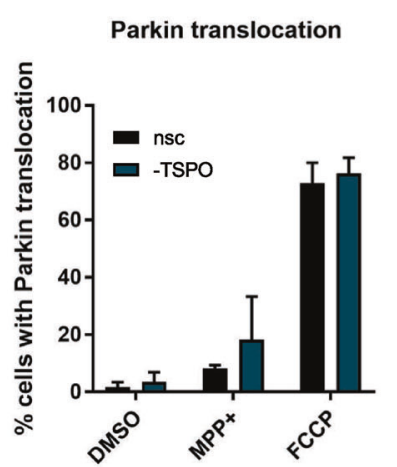

G

Ubiquitin-ATP5B co-localisation

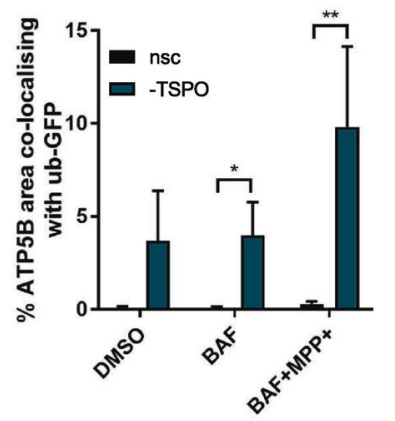

K

L

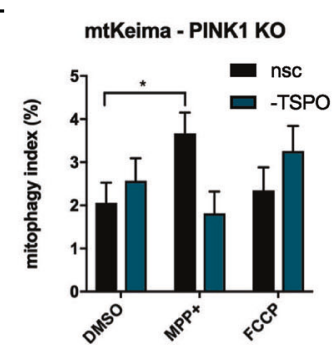

mitophagy pathway. Collectively, these data confirm a role for TSPO in basal and stress-induced mitochondrial quality control via de-ubiquitinating mechanisms. However, the necessity of utilising BAF to detect LC3 recruitment to mitochondria and $\mathrm{MPP}+$ induced ubiquitination implied a downstream effect on the autophagolysosomal pathway, which we therefore explored.

\section{Regulation of autophagolysosomal response by TSPO}

Using the live-cell imaging dye LysoTrackerTM DND-22 to mark lysosomes fluorescently, we assayed the lysosomal population in Control and -TSPO SH-SY5Y cells treated with $\mathrm{MPP}+(0.5 \mathrm{mM}, 20 \mathrm{~h})$ (Fig. 5A, B). The results 
4 Fig. 4 TSPO represses the MPP + mediated activation of mitophagy. A Mitophagy index measured with mtKeima in mock-transfected, \pm TSPO cells treated with vehicle (DMSO), $0.5 \mathrm{mM} \mathrm{MPP}+$ or $20 \mu \mathrm{M}$ FCCP for $20 \mathrm{~h}(n \geq 20$ cells). B ICC for ATP5B to label mitochondria (red) in cells transfected with TSPO siRNA/nsc and GFP-tagged LC3 and treated with either vehicle (DMSO), $10 \mathrm{nM}$ Bafilomycin 1A and $0.5 \mathrm{mM} \mathrm{MPP}+$ for $20 \mathrm{~h}$. The average number of mitochondrial LC3 punctae quantified in (C) ( $n \geq 10$ cells). D ICC analysis of Parkin translocation to mitochondria, immunostained for ATP5B. -TSPO cells transfected with Parkin-YFP, were treated for $20 \mathrm{~h}$ with DMSO, $0.5 \mathrm{mM}$ MPP + or $20 \mu \mathrm{M}$ FCCP. Percentage of cells with Parkin punctuation onto mitochondria ( $n \geq 20$ cells per condition) were represented in (E). F ICC for ATP5B to label mitochondria (red) in Control and -TSPO cells GFP-tagged ubiquitin and also treated with either vehicle (DMSO), $10 \mathrm{nM}$ Bafilomycin $1 \mathrm{~A}$ and $\mathrm{MPP}+(0.5 \mathrm{mM}$, $20 \mathrm{~h}$ ). G \% of mitochondria co-localised with ubiquitin punctae was used a measure of mitochondrial ubiquitination. $n \geq 10$ cells per condition. H Western blot of mock-transfected cells or overexpressing TSPO. Whole-cell protein lysates were probed for USP30 and a mitochondrial marker ATP5B. The blot was quantified in (I) $(n=3)$. $\mathbf{J}$ mRNA from Control and +TSPO cells was used for a quantitative reverse transcriptase PCR. The absolute mRNA copy number of USP30 was extrapolated and normalised to $18 \mathrm{~S}$ mRNA copy number. Each + TSPO value was then normalised to its Control $(n=3)$. K Representative WB bands of whole-cell protein lysates of WT and PINK1 KO SH-SY5Y cells probed for PINK1 and $\beta$ - actin (ACTB). $\mathbf{L}$ mtKeima measurement of mitophagy in nsc or - TSPO PINK1 KO cells treated with vehicle (DMSO), MPP $+(0.5 \mathrm{mM}, 20 \mathrm{~h})$ or FCCP

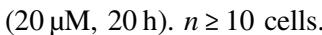

indicated that MPP + induces an increase in lysosomal counts in control cells, which reached values comparable to those registered in -TSPO cells at resting conditions. However, this effect was lost when the MEK-induced phosphorylation of ERK1/2 pathway was inhibited via UO126. Since the microphtalmia/trascription factor $\mathrm{E}$ (MiT/TFE) family of transcription factors comprises the most important regulators of lysosomal turnover [89], we sought to test their involvement, and by using ICC, we assayed the levels and subcellular localisation of transcription factor EB (TFEB) [90-92] (Fig. 5C, D). In our experimental system, we found that in -TSPO cells, the amount of nuclear TFEB increases when compared to control ones, and so does the number of lysosomes and their volume (Supplementary Fig. 2G, H). Being the expression of TSPO driven by the activation of the ERK pathway, which is engaged by MPP + [52] we explored whether the levels of TSPO affected the degree of pERK1/2 phosphorylation; the data in Fig. 5 panels E, F do confirm that TSPO does increase the degree of ERK1/2 phosphorylation. Having observed that MPP + and TSPO increase global ROS levels (Fig. 3), we sought to determine whether this effect could modulate ERK1/2 phosphorylation. Protein kinase C $\varepsilon$ (PKCE) is sensitive to redox status [93] and is known to phosphorylate ERK1/2 as well as to mediate phorbol myristate acetate (PMA)-induced TSPO upregulation [49, 94]. Therefore, TSPO may control its upstream regulator via a positive feedback mechanism, relying on oxidative stress. Furthermore, in keeping with this, the levels of pERK1/2 are dramatically reduced when TSPO-positive cells are treated with the antioxidant mitoTEMPO (Fig. 5G, H). In order to confirm this and corroborate the ample role played by TSPO on autophagy we mapped the transcriptome of dopaminergic neurons control (WT) versus those genetically depleted for TSPO (KO), untreated (UNT), or treated (MPP+) with the PD neurotoxin of interest. The next generation sequencing (NGS) analysis portraited a significant variance in the expression of the relative genes (Fig. 5I, J and Supplementary Fig. 4G, H) which was further evident among those which partake in general and selective autophagy (Fig. 5K). The working model depicted in Fig. 6 therefore proposes TSPO as required in the pathway to repress selection of mitochondria and therefore promote the aetiology of the whole network during neurotoxicity.

\section{Discussion}

The role of mitochondria in PD is known for nearly half of a century [4, 32, 95], but our understanding of their contribution to the disease's pathogenesis is unresolved. The mitochondrial decay in faulty dopaminergic neurons expands beyond the impairments in energy supply and second messengers (i.e. $\mathrm{Ca}^{2+}$ ) spanning inter-organelles communication and quality control mechanisms [7, 31, 96, 97]. This re-set the focus on mitochondrial aetiology of PD: 5 out of 18 risk factor genes for PD are linked to mitophagy (PARK2, PINK1, DJ-1, FBXO7, HTRA2). The incapacity of removing defective mitochondria through the process of mitophagy reroutes functional and architectural homeostasis of neurons to deficiencies. Finding early markers of mitochondrial commitment to PD is therefore pivotal to pave the way to improved diagnostic and therapeutic protocols.

In this work, we demonstrate TSPO to be one of those. Though enrolled to map neuroinflammation in several diseases [22, 98], little is known of TSPO molecular function in the pathogenesis of the conditions in which it is found upregulated [99, 100], and in which its ligands mediate neuroprotection [101]. In mice injected with MPTP (the pro-drug catalysed by MAO into MPP + [102]), treatment with XBD-173 (TSPO ligand) [103] preserves motor functions and striatal dopaminergic circuits [101]. Our data show that PD exploits TSPO to establish archetypical mitochondrial defects of the disease: increased redox stress (i), impaired mitophagy (ii), and susceptibility to dopamineinduced cell death (iii).

The heightened basal redox state in TSPO overexpressing neurons is not only due to the upregulation of the cytoplasmic NADPH oxidase [60], but also by the parallel accumulation of MAOB (Fig. 3H), which amplifies the presence of free radicals [104, 105]. 

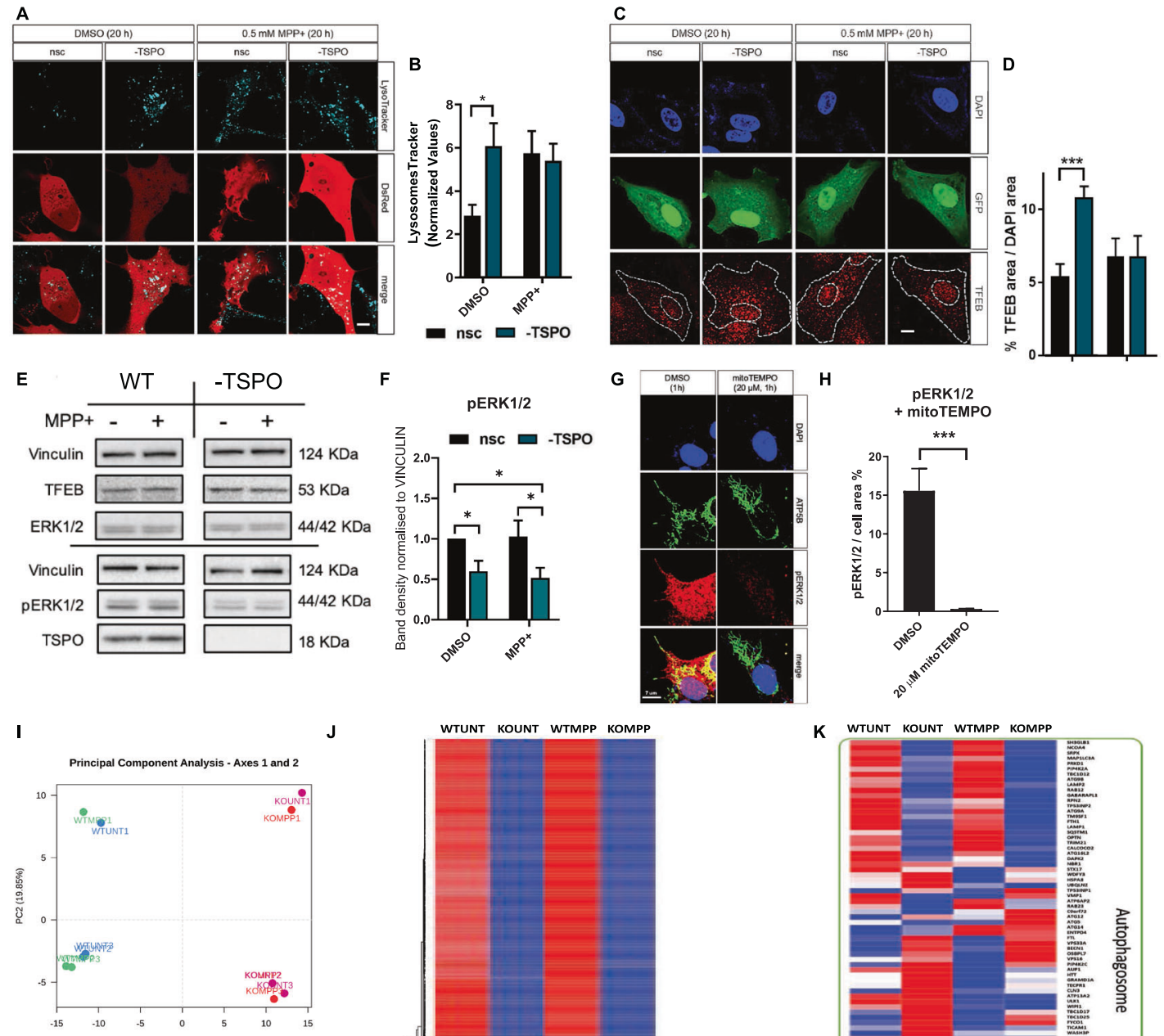

$J$
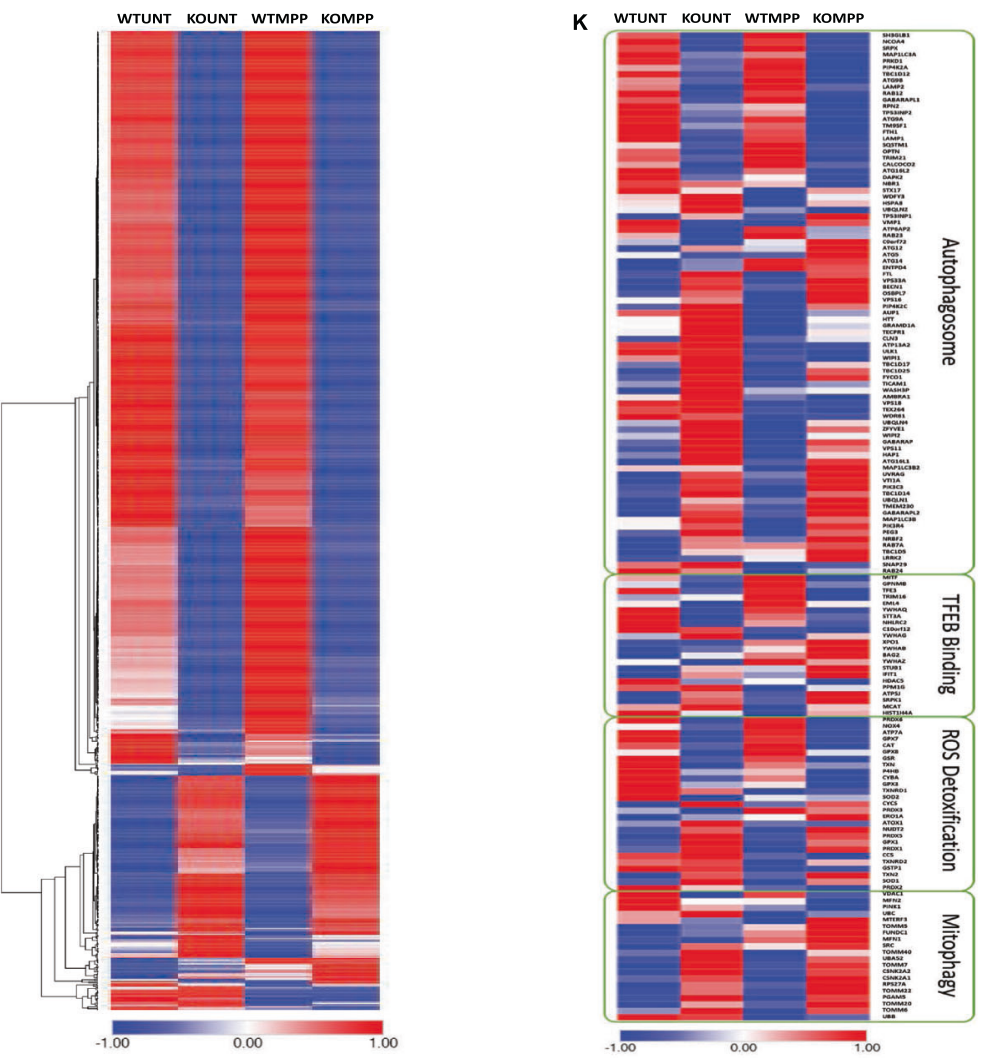

Fig. 5 Lysosomal homeostasis is impaired by TSPO. A Representative images of LysoTracker staining of the lysosomal population in cells control or -TSPO and treated with vehicle (DMSO), $0.5 \mathrm{mM}$ MPP + for $20 \mathrm{~h}$. B Quantification of the total lysosomal number per cell ( $n \geq 20$ cells). C Cells transfected with GFP control (nsc) and -TSPO were immunostained for TFEB following treatment with vehicle (DMSO), or $0.5 \mathrm{mM} \mathrm{MPP}+$ for $20 \mathrm{~h}$. D Quantification of TFEB nuclear localisation ( $n \geq 15$ cells). E Representative bands of Vinculin, TFEB, TSPO, ERK1/2, and pERK1/2 from WB SDS-PAGE of whole-cell lysates of Control (wt) and -TSPO cells. Average band density of $(\mathbf{F})$ pERK1/2 normalised to ERK1/2 and to respective Control $(n=3)$. Representative images of pERK1/2 ICC in wild type cells treated with $20 \mu \mathrm{M}$ mitoTEMPO (G). H Quantification of ERK1/ 2 phosphorylation via image-based analysis of pERK1/2 area over cell area $(n=20$ cells). I Principal component analysis of RNAseq data from Control and -TSPO cells. Heat maps in ( $\mathbf{J}$ and $\mathbf{K})$ are respectively of the full genome and genes involved in the cell's quality control regulation. 
Fig. 6 Model on TSPO role in the etiopathogenesis of neurotoxicity. The addition of PD neurotoxins like MPP + or 6-OHDA stimulates TSPO upregulation, which, by accumulating USP30 on mitochondria, impairs the organelle's ubiquitination mitophagy impairment. Concomitantly, it induces redox stress to promote ERK1/2 phosphorylation and modifications in the dynamics of the autophagolysosomal pathways.

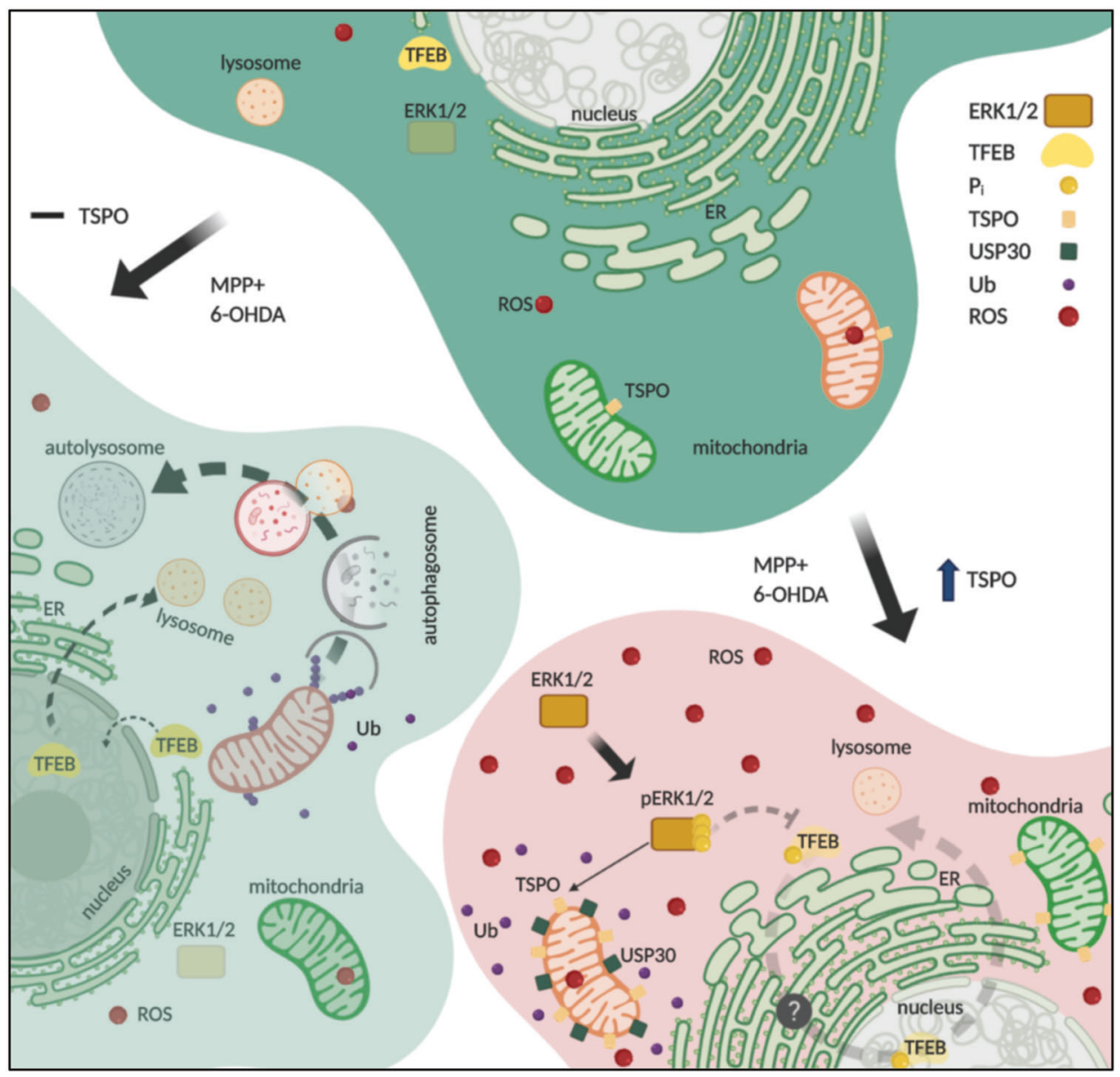

Interestingly, the absence of an MPP + induced spike of ROS in + TSPO cells indicates that neurons rich in TSPO retain higher levels of reduced GSH, which is consumed following MPP+ administration (Supplementary Fig. 1E-G). Therefore, we speculate that the excess of GSH reacts with the additional ROS preventing further and detrimental burst. In +TSPO cells, the greater GSH level may originate from the inhibition of Keap1, which would allow for the Nuclear factor erythroid 2-related factor 2 ( Nrf2) to migrate to the nucleus and upregulate the synthesis of GSH [106, 107]. The Nrf2 pathway is indeed responsible for the increased production of GSH s-transferase that executes the rate-limiting step in GSH production in the cytoplasm [108-110]. This was previously confirmed pharmacologically via the promitophagic drug PMI which represses TSPO [111].

The ability of TSPO to increase redox signalling while leaving unaltered the antioxidant pools would also explain why + TSPO cells resist cell death caused by a higher concentration of MPP + and excessive dopamine level (Fig. 2K, L) besides preventing the exacerbation of the MPP+induced oxidative stress (Fig. 3D, E, S). Accordingly, cells devoid of TSPO (-TSPO) undergo extensive $\Delta \Psi_{\mathrm{m}}$ depolarisation following treatment with MPP +
(Supplementary Fig. 2A-F), being at the same time more susceptible to death induced by the toxin (Fig. 2K) but not to the one caused by dopamine (Fig. 2L); indicative of an ongoing selection of defective mitochondria selection by mitophagy in these cells. And the analysis of mitochondrial morphology did reveal a more elongated and branched network of mitochondria in -TSPO cells corresponding to more favourable bioenergy $[112,113]$ as witnessed by the higher basal $\Delta \Psi_{\mathrm{m}}$ (Supplementary Fig. 2A-E).

Both 6-OHDA or MPP + trigger the MAPK/ERK pathway, which drives the expression of TSPO. This establishes a feedback loop for which ERK is continuously active -by the high degree of oxidative stress- repressing the retrotranslocation of TFEB to the nucleus and so the overall autophagy response by the repressed production of lysosomes (Figs. 4, 5).

Though deficiencies in lysosomal homeostasis could also be contributed by the impaired $\mathrm{Ca}^{2+}$ homeostasis driven by TSPO, the persistent level of free radicals of the oxygens in the cytosol (i) and the very nature of the trigger (MPP+) which engages the MAPK signalling (ii) suggest alterations in the TFEB transcriptional capacity to be the driving mechanism. 
Nuclear TFEB, an essential transcription factor for the completion of the autophagolysosomal pathway [90, 114], is upregulated in -TSPO cells and during challenge with PD toxins (Fig. 5). This made us propose that MPP + \{and therefore the neurotoxins capable of recapitulating the disease $[68,69,115]\}$ activate mitophagy by stimulating TFEB, which is negatively regulated by the concomitant overexpression of TSPO (Fig. 5). The transcriptome data well corroborate that loss of TSPO in neurons is pivotal to several genetic alterations, which are exploited further by the treatment with the neurotoxin MPP+ (Fig. 5 and Supplementary Fig. 4G, H). The limited availability of autophagosomes and production of lysosomes (Fig. 5) aligns with the deubiquitylation of mitochondria perpetrated by TSPO.

Mitophagy occurs if de-ubiquitinating molecules such as USP30 are inhibited [88]. TSPO, on the contrary, stabilises USP30, thereby impeding the processing of mitochondrial proteins required for the completion of the pathway (Fig. 4H, I). USP30 has been considered a therapeutic target since the discovery of its involvement in mitophagy [46], whereas its inhibition rescues motor symptoms and lifespan of PD Drosophila models. The recent publications of three proteomic studies $[44,45,116]$ have highlighted that changes in the ubiquitylome at appropriate dosages faithfully replicate the genetic ablation of USP30, which nonetheless remains particularly hostile to target with chemicals. TSPO could therefore offer an alternative site for therapeutic inferring of USP30, and advantages for this approach stem from the extensively studied pharmacodynamic and pharmacokinetic properties of its ligands [17, 20, 117].

It is known that the monogenic forms of the disease constitute only $5-10 \%$ of the total PD cases [118, 119], demanding for a greater understanding of the aetiological factors at the basis of the sporadic forms, which account for the greatest portion of cases. TSPO merges as a potential target to inform and control both onset and penetrance. Though its anti-mitophagic role will intensify the retrocommunication with nucleus [120] to favour cell survival, this beneficial effect will be short-lived as TSPO-positive neurons will succumb to dopamine toxicity in the long-term (Fig. 2L and Supplementary Fig. 4A-F).

Importantly the upregulation of TSPO in response to neurotoxins is selective for neurons as microglial cells are unresponsive to PD toxins (Supplementary Fig. 3A, B).

In light of these data, it is not surprising that direct detection of the protein in mice and in vivo PET measurements demonstrate higher TSPO expression with aging $[60,121]$, which is per se a demonstrated risk factor for the development and progression of Parkinson's disease (PD).

Acknowledgements The research activities lead by MC are supported by the following funders: Biotechnology and Biological Sciences Research Council [grant numbers BB/M010384/1 and BB/N007042/1];
BBSRC LiDO Studentship Scheme; Umberto Veronesi Foundation Young Investigator Award Programme; Marie Curie CIG 304165 _TSPO \& Brain; LAM-Bighi Grant Initiative; The Rotary Foundation and the European Research Council COG -819600_FIRM. The Parkinson's UK (grant F1301) for HM and the National Institute for Health Research and Hospitals Biomedical Research Centre UCLH for support to PG and RA should be also mentioned.

\section{Compliance with ethical standards}

Conflict of interest The authors declare no competing interests.

Publisher's note Springer Nature remains neutral with regard to jurisdictional claims in published maps and institutional affiliations.

Open Access This article is licensed under a Creative Commons Attribution 4.0 International License, which permits use, sharing, adaptation, distribution and reproduction in any medium or format, as long as you give appropriate credit to the original author(s) and the source, provide a link to the Creative Commons license, and indicate if changes were made. The images or other third party material in this article are included in the article's Creative Commons license, unless indicated otherwise in a credit line to the material. If material is not included in the article's Creative Commons license and your intended use is not permitted by statutory regulation or exceeds the permitted use, you will need to obtain permission directly from the copyright holder. To view a copy of this license, visit http://creativecommons. org/licenses/by/4.0/.

\section{References}

1. Inczedy-Farkas G, Remenyi V, Gal A, Varga Z, Balla P, Udvardy-Meszaros A, et al. Psychiatric symptoms of patients with primary mitochondrial DNA disorders. Behav Brain Funct BBF. 2012;8:9.

2. Anglin RE, Garside SL, Tarnopolsky MA, Mazurek MF, Rosebush PI. The psychiatric manifestations of mitochondrial disorders: a case and review of the literature. J Clin Psychiatry. 2012;73:506-12.

3. Gunnewiek TMK, Hugte EJHV, Frega M, Guardia GS, Foreman K, Panneman D. m.3243A > G-Induced Mitochondrial Dysfunction Impairs Human Neuronal Development and Reduces Neuronal Network Activity and Synchronicity. Cell Rep. 2020;31:107538.

4. Shulman JM, De Jager PL, Feany MB. Parkinson's disease: genetics and pathogenesis. Annu Rev Pathol. 2011;6:193-222.

5. de Lau LML, Breteler MMB. Epidemiology of Parkinson's disease. Lancet Neurol. 2006;5:525-35.

6. Martin I, Dawson VL, Dawson TM. Recent advances in the genetics of Parkinson's disease. Annu Rev Genomics Hum Genet. 2011;12:301-25.

7. Pickrell AM, Youle RJ. The roles of PINK1, parkin, and mitochondrial fidelity in Parkinson's disease. Neuron. 2015;85:257-73.

8. Braestrup C, Albrechtsen R, Squires RF. High densities of benzodiazepine receptors in human cortical areas. Nature. 1977;269:702-4.

9. Braestrup C, Squires RF. Specific benzodiazepine receptors in rat brain characterized by high-affinity $(3 \mathrm{H})$ diazepam binding. Proc Natl Acad Sci USA. 1977;74:3805-9.

10. Rupprecht R, Rammes G, Eser D, Baghai TC, Schüle C, Nothdurfter $\mathrm{C}$, et al. Translocator protein $(18 \mathrm{kD})$ as target for anxiolytics without benzodiazepine-like side effects. Science. 2009;325:490-3. 
11. Rupprecht R, Papadopoulos V, Rammes G, Baghai TC, Fan J, Akula N, et al. Translocator protein (18 kDa) (TSPO) as a therapeutic target for neurological and psychiatric disorders. Nat Rev Drug Discov. 2010;9:971-88.

12. Gerhard A. TSPO imaging in parkinsonian disorders. Clin Transl Imaging. 2016;4:183-90.

13. Iannaccone S, Cerami C, Alessio M, Garibotto V, Panzacchi A, Olivieri S, et al. In vivo microglia activation in very early dementia with Lewy bodies, comparison with Parkinson's disease. Parkinsonism Relat Disord. 2013;19:47-52.

14. Ishizawa K, Dickson DW. Microglial activation parallels system degeneration in progressive supranuclear palsy and corticobasal degeneration. J Neuropathol Exp Neurol. 2001;60:647-57.

15. Anholt RR, De Souza EB, Oster-Granite ML, Snyder SH. Peripheral-type benzodiazepine receptors: autoradiographic localization in whole-body sections of neonatal rats. J Pharmacol Exp Ther. 1985;233:517-26.

16. Banati RB, Middleton RJ, Chan R, Hatty CR, Kam WW-Y, Quin $\mathrm{C}$, et al. Positron emission tomography and functional characterization of a complete PBR/TSPO knockout. Nat Commun. 2014;5:5452.

17. Banati RB. Visualising microglial activation in vivo. Glia. 2002;40:206-17.

18. Cosenza-Nashat M, Zhao M-L, Suh H-S, Morgan J, Natividad R, Morgello S, et al. Expression of the translocator protein of 18 $\mathrm{kDa}$ by microglia, macrophages and astrocytes based on immunohistochemical localization in abnormal human brain. Neuropathol Appl Neurobiol. 2009;35:306-28.

19. Hellberg S, Silvola JMU, Kiugel M, Liljenbäck H, Savisto N, Li $\mathrm{X}-\mathrm{G}$, et al. 18-kDa translocator protein ligand (18)F-FEMPA: Biodistribution and uptake into atherosclerotic plaques in mice. $\mathrm{J}$ Nucl Cardiol Off Publ Am Soc Nucl Cardiol. 2016;25 (May):2016 https://doi.org/10.1007/s12350-016-0527-y.

20. Cagnin A, Kassiou M, Meikle SR, Banati RB. Positron emission tomography imaging of neuroinflammation. Neurother J Am Soc Exp Neurother. 2007;4:443-52.

21. Liu G-J, Middleton RJ, Hatty CR, Kam WW-Y, Chan R, Pham $\mathrm{T}$, et al. The $18 \mathrm{kDa}$ translocator protein, microglia and neuroinflammation. Brain Pathol Zurich Switz. 2014;24:631-53.

22. Belloli S, Morari M, Murtaj V, Valtorta S, Moresco RM, Gilardi MC. Translation Imaging in Parkinson's Disease: focus on Neuroinflammation. Front Aging Neurosci. 2020;12:152.

23. Mills CD, Bitler JL, Woolf CJ. Role of the peripheral benzodiazepine receptor in sensory neuron regeneration. Mol Cell Neurosci. 2005;30:228-37.

24. Notter T, Schalbetter SM, Clifton NE, Mattei D, Richetto J, Thomas K, et al. Neuronal activity increases translocator protein (TSPO) levels. Mol Psychiatry. 2020:12.

25. Palzur E, Sharon A, Shehadeh M, Soustiel JF. Investigation of the mechanisms of neuroprotection mediated by Ro5-4864 in brain injury. Neuroscience. 2016;329:162-70.

26. Anholt RR, Murphy KM, Mack GE, Snyder SH. Peripheral-type benzodiazepine receptors in the central nervous system: localization to olfactory nerves. J Neurosci Off. J Soc Neurosci. 1984;4:593-603.

27. Karchewski LA, Bloechlinger S, Woolf CJ. Axonal injurydependent induction of the peripheral benzodiazepine receptor in small-diameter adult rat primary sensory neurons. Eur J Neurosci. 2004;20:671-83.

28. Benavides J, Quarteronet D, Imbault F, Malgouris C, Uzan A, Renault C, et al. Labelling of 'peripheral-type' benzodiazepine binding sites in the rat brain by using [3H]PK 11195 , an isoquinoline carboxamide derivative: kinetic studies and autoradiographic localization. J Neurochem. 1983;41:1744-50.

29. Walker MD, Dinelle K, Kornelsen R, Lee NV, Miao Q, Adam $\mathrm{M}$, et al. [11C]PBR28 PET imaging is sensitive to neuroinflammation in the aged rat. $\mathrm{J}$ Cereb Blood Flow Metab. 2015;35:1331-8.

30. Gatliff J, East D, Crosby J, Abeti R, Harvey R, Craigen W, et al. TSPO interacts with VDAC1 and triggers a ROS-mediated inhibition of mitochondrial quality control. 2014;10:2279-96.

31. Bender A, Krishnan KJ, Morris CM, Taylor GA, Reeve AK, Perry RH, et al. High levels of mitochondrial DNA deletions in substantia nigra neurons in aging and Parkinson disease. Nat Genet. 2006;38:515-7.

32. Schapira AH, Cooper JM, Dexter D, Clark JB, Jenner P, Marsden CD. Mitochondrial complex I deficiency in Parkinson's disease. J Neurochem. 1990;54:823-7.

33. Matic I, Strobbe D, Frison M, Campanella M. Controlled and impaired mitochondrial quality in neurons: molecular Physiology and prospective Pharmacology. Pharmacol Res Off J Ital Pharmacol Soc. 2015;23(April):2015 https://doi.org/10.1016/j.phrs. 2015.03.021.

34. Kageyama Y, Zhang Z, Roda R, Fukaya M, Wakabayashi J, Wakabayashi N, et al. Mitochondrial division ensures the survival of postmitotic neurons by suppressing oxidative damage. J Cell Biol. 2012;197:535-51.

35. Valente EM, Abou-Sleiman PM, Caputo V, Muqit MMK, Harvey $\mathrm{K}$, Gispert S, et al. Hereditary early-onset Parkinson's disease caused by mutations in PINK1. Science. 2004;304:1158-60.

36. Kitada T, Asakawa S, Hattori N, Matsumine H, Yamamura Y, Minoshima S, et al. Mutations in the parkin gene cause autosomal recessive juvenile Parkinsonism. Nature. 1998;392:605-8.

37. Narendra D, Tanaka A, Suen D-F, Youle RJ. Parkin is recruited selectively to impaired mitochondria and promotes their autophagy. J Cell Biol. 2008;183:795-803.

38. Lazarou M, Sliter DA, Kane LA, Sarraf SA, Wang C, Burman $\mathrm{JL}$, et al. The ubiquitin kinase PINK1 recruits autophagy receptors to induce mitophagy. Nature. 2015;524:309-14.

39. Poole AC, Thomas RE, Andrews LA, McBride HM, Whitworth AJ, Pallanck LJ. The PINK1/Parkin pathway regulates mitochondrial morphology. Proc Natl Acad Sci. 2008;105:1638-43.

40. McWilliams TG, Prescott AR, Montava-Garriga L, Ball G, Singh F, Barini E, et al. Basal Mitophagy Occurs Independently of PINK1 in Mouse Tissues of High Metabolic Demand. Cell Metab. 2018;10 (January):2018 https://doi.org/10.1016/j.cmet.2017.12.008.

41. Lee JJ, Sanchez-Martinez A, Zarate AM, Benincá C, Mayor U, Clague MJ, et al. Basal mitophagy is widespread in Drosophila but minimally affected by loss of Pink1 or parkin. J Cell Biol. 2018:jcb.201801044.

42. Sliter DA, Martinez J, Hao L, Chen X, Sun N, Fischer TD, et al. Parkin and PINK1 mitigate STING-induced inflammation. Nature. 2018;561:258-62.

43. Matheoud D, Cannon T, Voisin A, Penttinen A-M, Ramet L, Fahmy AM, et al. Intestinal infection triggers Parkinson's disease-like symptoms in Pink1-/- mice. Nature. 2019;571: $565-9$.

44. Ordureau A, Paulo JA, Zhang J, An H, Swatek KN, Cannon JR, et al. Global Landscape and Dynamics of Parkin and USP30Dependent Ubiquitylomes in iNeurons during Mitophagic Signaling. Mol Cell. 2020;77:1124. e10

45. Phu L, Rose CM, Tea JS, Wall CE, Verschueren E, Cheung TK, et al. Dynamic Regulation of Mitochondrial Import by the Ubiquitin System. Mol Cell. 2020;77:1107. e10

46. Bingol B, Tea JS, Phu L, Reichelt M, Bakalarski CE, Song Q, et al. The mitochondrial deubiquitinase USP30 opposes parkinmediated mitophagy. Nature. 2014;510:370-5.

47. Harper JW, Ordureau A, Heo J-M. Building and decoding ubiquitin chains for mitophagy. Nat Rev Mol Cell Biol. 2018;19:93.

48. Farré J-C, Subramani S. Mechanistic insights into selective autophagy pathways: lessons from yeast. Nat Rev Mol Cell Biol. 2016;17:537-52. 
49. Batarseh A, Li J, Papadopoulos V. Protein kinase C epsilon regulation of translocator protein $(18 \mathrm{kDa})$ Tspo gene expression is mediated through a MAPK pathway targeting STAT3 and cJun transcription factors. Biochemistry. 2010;49:4766-78.

50. Cruz CD, Cruz F. The ERK 1 and 2 Pathway in the Nervous System: From Basic Aspects to Possible Clinical Applications in Pain and Visceral Dysfunction. Curr Neuropharmacol. 2007;5:244-52.

51. Zhu J-H, Guo F, Shelburne J, Watkins S, Chu CT. Localization of Phosphorylated ERK/MAP Kinases to Mitochondria and Autophagosomes in Lewy Body Diseases. Brain Pathol. 2003;13:473-81.

52. Zhu JH, Gusdon AM, Cimen H, Van Houten B, Koc E, Chu CT. Impaired mitochondrial biogenesis contributes to depletion of functional mitochondria in chronic MPP+ toxicity: dual roles for ERK1/2. Cell Death Dis. 2012;3:e312.

53. Wang KZQ, Zhu J, Dagda RK, Uechi G, Cherra SJ III, Gusdon AM, et al. ERK-mediated phosphorylation of TFAM downregulates mitochondrial transcription: Implications for Parkinson's disease. Mitochondrion. 2014;17:132-40.

54. Duncan OF, Granat L, Ranganathan R, Singh VK, Mazaud D, Fanto M, et al. Ras-ERK-ETS inhibition alleviates neuronal mitochondrial dysfunction by reprogramming mitochondrial retrograde signaling. PLOS Genet. 2018;14:e1007567.

55. Westphal R, Sumiyoshi A, Simmons C, Mesquita M, Wood TC, Williams SCR, et al. Characterization of gray matter atrophy following 6-hydroxydopamine lesion of the nigrostriatal system. Neuroscience. 2016;334:166-79.

56. Paxinos G, Watson C. The Rat Brain in Stereotaxic Coordinates. 1st ed. New York: Academic Press; 1986.

57. Datla KP, Zbarsky V, Dexter DT. Effects of anaesthetics on the loss of nigrostriatal dopaminergic neurons by 6-hydroxydopamine in rats. J Neural Transm. 2006;113:583-91.

58. Morelli A, Chiozzi P, Chiesa A, Ferrari D, Sanz JM, Falzoni S, et al. Extracellular ATP causes ROCK I-dependent bleb formation in P2X7-transfected HEK293 cells. Mol Biol Cell. 2003;14:2655-64.

59. Mortiboys H, Thomas KJ, Koopman WJH, Klaffke S, AbouSleiman P, Olpin S, et al. Mitochondrial function and morphology are impaired in parkin-mutant fibroblasts. Ann Neurol. 2008;64:555-65.

60. Gatliff J, East DA, Singh A, Alvarez MS, Frison M, Matic I, et al. A role for TSPO in mitochondrial $\mathrm{Ca} 2+$ homeostasis and redox stress signaling. Cell Death Dis. 2017;8:e2896.

61. Gentleman RC, Carey VJ, Bates DM, Bolstad B, Dettling M, Dudoit $\mathrm{S}$, et al. Bioconductor: open software development for computational biology and bioinformatics. Genome Biol. 2004; 5:R80.

62. Love MI, Huber W, Anders S. Moderated estimation of fold change and dispersion for RNA-seq data with DESeq2. Genome Biol. 2014;15:550.

63. Alhamdoosh M, Ng M, Wilson NJ, Sheridan JM, Huynh H, Wilson MJ, et al. Combining multiple tools outperforms individual methods in gene set enrichment analyses. Bioinforma Oxf Engl. 2017;33:414-24.

64. Zhang Y, Chen K, Sloan SA, Bennett ML, Scholze AR, O'Keeffe S, et al. An RNA-Sequencing Transcriptome and Splicing Database of Glia, Neurons, and Vascular Cells of the Cerebral Cortex. J Neurosci. 2014;34:11929-47.

65. Politis M. Neuroimaging in Parkinson disease: from research setting to clinical practice. Nat Rev Neurol. 2014;10:708-22.

66. Kitayama S, Mitsuhata C, Davis S, Wang JB, Sato T, Morita K, et al. MPP+ toxicity and plasma membrane dopamine transporter: study using cell lines expressing the wild-type and mutant rat dopamine transporters. Biochim Biophys Acta. 1998;1404:305-13.

67. Lavalaye J, Booij J, Reneman L, Habraken JB, van Royen EA. Effect of age and gender on dopamine transporter imaging with
[123I]FP-CIT SPET in healthy volunteers. Eur J Nucl Med. 2000;27:867-9.

68. Betarbet R, Sherer TB, MacKenzie G, Garcia-Osuna M, Panov AV, Greenamyre JT. Chronic systemic pesticide exposure reproduces features of Parkinson's disease. Nat Neurosci. 2000;3:1301-6.

69. Javitch JA, D'Amato RJ, Strittmatter SM, Snyder SH. Parkinsonism-inducing neurotoxin, N-methyl-4-phenyl-1,2,3,6 -tetrahydropyridine: uptake of the metabolite N-methyl-4phenylpyridine by dopamine neurons explains selective toxicity. Proc Natl Acad Sci USA. 1985;82:2173-7.

70. Zhu J-H, Horbinski C, Guo F, Watkins S, Uchiyama Y, Chu CT. Regulation of autophagy by extracellular signal-regulated protein kinases during 1-methyl-4-phenylpyridinium-induced cell death. Am J Pathol. 2007;170:75-86.

71. Gao H, Yang W, Qi Z, Lu L, Duan C, Zhao C, et al. DJ-1 Protects Dopaminergic Neurons against Rotenone-Induced Apoptosis by Enhancing ERK-Dependent Mitophagy. J Mol Biol. 2012;423:232-48.

72. Giordano S, Lee J, Darley-Usmar VM, Zhang J. Distinct effects of rotenone, 1-methyl-4-phenylpyridinium and 6hydroxydopamine on cellular bioenergetics and cell death. PloS ONE. 2012;7:e44610.

73. Packer MA, Miesel R, Murphy MP. Exposure to the parkinsonian neurotoxin 1-methyl-4-phenylpyridinium (MPP+) and nitric oxide simultaneously causes cyclosporin A-sensitive mitochondrial calcium efflux and depolarisation. Biochem Pharmacol. 1996;51:267-73.

74. Miyako K, Irie T, Muta T, Umeda S, Kai Y, Fujiwara T, et al. 1Methyl-4-phenylpyridinium ion (MPP+) selectively inhibits the replication of mitochondrial DNA. Eur J Biochem. 1999;259: 412-8.

75. Murphy MP, Krueger MJ, Sablin SO, Ramsay RR, Singer TP. Inhibition of complex I by hydrophobic analogues of N-methyl4-phenylpyridinium (MPP+) and the use of an ion-selective electrode to measure their accumulation by mitochondria and electron-transport particles. Biochem J. 1995;306:359-65.

76. Frison M, Mallach AK, Kennedy E, Campanella M. The $18 \mathrm{kDa}$ Translocator Protein (TSPO): Cholesterol Trafficking and the Biology of a Prognostic and Therapeutic Mitochondrial Target. Mol Basis Mitochondrial Signal. Springer, Cham. 2017;285-315

77. McEnery MW, Snowman AM, Trifiletti RR, Snyder SH. Isolation of the mitochondrial benzodiazepine receptor: association with the voltage-dependent anion channel and the adenine nucleotide carrier. Proc Natl Acad Sci USA. 1992;89:3170-4.

78. Guilarte TR, Loth MK, Guariglia SR. TSPO Finds NOX2 in Microglia for Redox Homeostasis. Trends Pharmacol Sci. 2016;29(March):2016 https://doi.org/10.1016/j.tips.2016.02.008.

79. Shih JC, Wu JB, Chen K. Transcriptional regulation and multiple functions of MAO genes. J Neural Transm. 2011;118:979-86.

80. Ekblom J, Jossan SS, Oreland L, Walum E, Aquilonius S-M. Reactive gliosis and monoamine oxidase B. In: Tipton KF, Youdim MBH, Barwell CJ, Callingham BA, Lyles GA, editors. Amine Oxidases Funct. Dysfunct., Vienna: Springer; 1994. pp. 253-8.

81. Mallajosyula JK, Kaur D, Chinta SJ, Rajagopalan S, Rane A, Nicholls DG, et al. MAO-B Elevation in Mouse Brain Astrocytes Results in Parkinson's Pathology. PLoS ONE. 2008;3:e1616.

82. Georgakopoulos ND, Wells G, Campanella M. The pharmacological regulation of cellular mitophagy. Nat Chem Biol. 2017;13:136-46.

83. Palikaras K, Lionaki E, Tavernarakis N. Mechanisms of mitophagy in cellular homeostasis, physiology and pathology. Nat Cell Biol. 2018;20:1013-22.

84. Dagda RK, Zhu J, Kulich SM, Chu CT. Mitochondrially localized ERK2 regulates mitophagy and autophagic cell stress: 
implications for Parkinson's disease. Autophagy. 2008;4: 770-82.

85. Chu CT, Ji J, Dagda RK, Jiang JF, Tyurina YY, Kapralov AA, et al. Cardiolipin externalization to the outer mitochondrial membrane acts as an elimination signal for mitophagy in neuronal cells. Nat Cell Biol. 2013;15:1197-205.

86. Yoshimori T, Yamamoto A, Moriyama Y, Futai M, Tashiro Y. Bafilomycin A1, a specific inhibitor of vacuolar-type $\mathrm{H}$ $(+)$-ATPase, inhibits acidification and protein degradation in lysosomes of cultured cells. J Biol Chem. 1991;266:17707-12.

87. Komander D, Clague MJ, Urbé S. Breaking the chains: structure and function of the deubiquitinases. Nat Rev Mol Cell Biol. 2009;10:550-63.

88. Marcassa E, Kallinos A, Jardine J, Rusilowicz-Jones EV, Martinez A, Kuehl S, et al. Dual role of USP30 in controlling basal pexophagy and mitophagy. EMBO Rep. 2018;19:e45595.

89. Palmieri M, Impey S, Kang H, di Ronza A, Pelz C, Sardiello M, et al. Characterization of the CLEAR network reveals an integrated control of cellular clearance pathways. Hum Mol Genet. 2011;20:3852-66.

90. Settembre C, Malta CD, Polito VA, Arencibia MG, Vetrini F, Erdin S, et al. TFEB Links Autophagy to Lysosomal Biogenesis. Science. 2011;332:1429-33.

91. Settembre C, Zoncu R, Medina DL, Vetrini F, Erdin S, Erdin S, et al. A lysosome-to-nucleus signalling mechanism senses and regulates the lysosome via mTOR and TFEB. EMBO J. 2012;31:1095-108.

92. Palmieri M, Pal R, Nelvagal HR, Lotfi P, Stinnett GR, Seymour $\mathrm{ML}$, et al. mTORC1-independent TFEB activation via Akt inhibition promotes cellular clearance in neurodegenerative storage diseases. Nat Commun. 2017;8:14338.

93. Cosentino-Gomes D, Rocco-Machado N, Meyer-Fernandes JR. Cell Signaling through Protein Kinase C Oxidation and Activation. Int J Mol Sci. 2012;13:10697-721.

94. Batarseh A, Giatzakis C, Papadopoulos V. Phorbol-12-myristate 13-acetate acting through protein kinase Cepsilon induces translocator protein $(18-\mathrm{kDa})$ TSPO gene expression. Biochemistry. 2008;47:12886-99.

95. Mizuno Y, Suzuki K, Sone N, Saitoh T. Inhibition of ATP synthesis by 1-methyl-4-phenylpyridinium ion (MPP+) in isolated mitochondria from mouse brains. Neurosci Lett. 1987;81:204-8.

96. Wu W, Lin C, Wu K, Jiang L, Wang X, Li W, et al. FUNDC1 regulates mitochondrial dynamics at the ER-mitochondrial contact site under hypoxic conditions. EMBO J. 2016;35:1368-84.

97. Lackner LL. The Expanding and Unexpected Functions of Mitochondria Contact Sites. Trends Cell Biol. 2019;29:580-90.

98. London ED, Okita K, Kinney KR, Dean AC, McClintick MN, Rizor EJ, et al. No significant elevation of translocator protein binding in the brains of recently abstinent methamphetamine users. Drug Alcohol Depend. 2020;213:108104.

99. Gut P, Zweckstetter M, Banati RB. Lost in translocation: the functions of the $18-\mathrm{kD}$ translocator protein. Trends Endocrinol Metab TEM. 2015;26:349-56.

100. Selvaraj V, Stocco DM. The changing landscape in translocator protein (TSPO) function. Trends Endocrinol Metab. 2015;26:341-8.

101. Gong J, Szego ÉM, Leonov A, Benito E, Becker S, Fischer A, et al. Translocator Protein Ligand Protects against Neurodegeneration in the MPTP Mouse Model of Parkinsonism. J Neurosci Off. J Soc Neurosci. 2019;39:3752-69.

102. Chiba K, Trevor A, Castagnoli N. Metabolism of the neurotoxic tertiary amine, MPTP, by brain monoamine oxidase. Biochem Biophys Res Commun. 1984;120:574-8.
103. Strobbe D, Campanella M. Anxiolytic Therapy: a Paradigm of Successful Mitochondrial Pharmacology. Trends Pharmacol Sci. 2018;30(March):2018 https://doi.org/10.1016/j.tips.2018.02.008.

104. Youdim MBH, Edmondson D, Tipton KF. The therapeutic potential of monoamine oxidase inhibitors. Nat Rev Neurosci. 2006;7:295-309.

105. LeWitt PA. Clinical trials of neuroprotection in Parkinson's disease: long-term selegiline and alpha-tocopherol treatment. J Neural Transm Suppl. 1994;43:171-81.

106. Hayes JD, McMahon M, Chowdhry S, Dinkova-Kostova AT. Cancer chemoprevention mechanisms mediated through the Keap1-Nrf2 pathway. Antioxid Redox Signal. 2010;13: 1713-48.

107. Magesh S, Chen Y, Hu L. Small molecule modulators of Keap1Nrf2-ARE pathway as potential preventive and therapeutic agents. Med Res Rev. 2012;32:687-726.

108. Harvey CJ, Thimmulappa RK, Singh A, Blake DJ, Ling G, Wakabayashi $\mathrm{N}$, et al. Nrf2-regulated glutathione recycling independent of biosynthesis is critical for cell survival during oxidative stress. Free Radic Biol Med. 2009;46:443-53.

109. Lu SC. Regulation of Glutathione Synthesis. Mol Aspects Med. 2009;30:42-59.

110. Marí M, Morales A, Colell A, García-Ruiz C, Fernández-Checa JC. Mitochondrial Glutathione, a Key Survival Antioxidant. Antioxid Redox Signal. 2009;11:2685-2700.

111. Georgakopoulos ND, Frison M, Alvarez MS, Bertrand H, Wells G, Campanella M. Reversible Keap1 inhibitors are preferential pharmacological tools to modulate cellular mitophagy. Sci Rep. 2017;7:10303.

112. Ni H-M, Williams JA, Ding W-X. Mitochondrial dynamics and mitochondrial quality control. Redox Biol. 2015;4:6-13.

113. Tilokani L, Nagashima S, Paupe V, Prudent J. Mitochondrial dynamics: overview of molecular mechanisms. Essays Biochem. 2018;62:341-60.

114. Luzio JP, Hackmann Y, Dieckmann NMG, Griffiths GM. The Biogenesis of Lysosomes and Lysosome-Related Organelles. Cold Spring Harb Perspect Biol. 2014;6:a016840.

115. Simola N, Morelli M, Carta AR. The 6-Hydroxydopamine model of parkinson's disease. Neurotox Res. 2007;11:151-67.

116. Rusilowicz-Jones E, Jardine J, Kallinos A, Pinto-Fernandez A, Guenther F, Giurrandino M, et al. A novel USP30 inhibitor recapitulates genetic loss of USP30 and sets the trigger for PINK1-PARKIN amplification of mitochondrial ubiquitylation. BioRxiv. 2020:2020.04.16.044206.

117. Turkheimer FE, Rizzo G, Bloomfield PS, Howes O, ZanottiFregonara P, Bertoldo A, et al. The methodology of TSPO imaging with positron emission tomography. Biochem Soc Trans. 2015;43:586-92.

118. Alcalay R, Caccappolo E, Mejia-Santana H, Tang M-X, Rosado L, Ross B, et al. Frequency of known mutations in early onset PD; implication for genetic counseling: the CORE-PD study. Arch Neurol. 2010;67:1116-22.

119. Klein C, Westenberger A. Genetics of Parkinson's Disease. Cold Spring Harb Perspect Med. 2012;2:a008888.

120. Desai R, East DA, Hardy L, Crosby J, Rigon M, Faccenda D, et al. Mitochondria form contact sites with the nucleus to couple prosurvival retrograde response. Sci Adv. 2020;6(Dec 18): eabc9955.

121. Kumar A, Muzik O, Shandal V, Chugani D, Chakraborty P, Chugani HT. Evaluation of age-related changes in translocator protein (TSPO) in human brain using (11)C-[R]-PK11195 PET. J Neuroinflammation. 2012;9:232. 